\title{
Sustained mitogen-activated protein kinase activation reprograms defense metabolism and phosphoprotein profile in Arabidopsis thaliana
}

\author{
Ines Lassowskat ${ }^{1}$, Christoph Böttcher ${ }^{1,2}$, Lennart Eschen-Lippold ${ }^{1}$, Dierk Scheel $^{1}$ and Justin Lee ${ }^{1 *}$ \\ ${ }^{1}$ Department of Stress and Developmental Biology, Leibniz Institute of Plant Biochemistry, Halle/Saale, Germany \\ ${ }^{2}$ Federal Research Centre for Cultivated Plants, Institute for Ecological Chemistry, Plant Analysis and Stored Product Protection, Julius Kühn Institute, Berlin, \\ Germany
}

\section{Edited by:}

Dominique Job, Centre National de la Recherche Scientifique, France

\section{Reviewed by:}

Stefanie Wienkoop, University of Vienna, Austria

Hans-Peter Mock, Institute of Plant Genetics and Crop Plant Research (IPK), Germany

\section{*Correspondence:}

Justin Lee, Department of Stress and Developmental Biology, Leibniz Institute of Plant Biochemistry,

Weinberg 3, D-06120 Halle/Saale,

Germany

e-mail: jlee@ipb-halle.de
Mitogen-activated protein kinases (MAPKs) target a variety of protein substrates to regulate cellular signaling processes in eukaryotes. In plants, the number of identified MAPK substrates that control plant defense responses is still limited. Here, we generated transgenic Arabidopsis thaliana plants with an inducible system to simulate in vivo activation of two stress-activated MAPKs, MPK3, and MPK6. Metabolome analysis revealed that this artificial MPK3/6 activation (without any exposure to pathogens or other stresses) is sufficient to drive the production of major defense-related metabolites, including various camalexin, indole glucosinolate and agmatine derivatives. An accompanying (phospho)proteome analysis led to detection of hundreds of potential phosphoproteins downstream of MPK3/6 activation. Besides known MAPK substrates, many candidates on this list possess typical MAPK-targeted phosphosites and in many cases, the corresponding phosphopeptides were detected by mass spectrometry. Notably, several of these putative phosphoproteins have been reported to be associated with the biosynthesis of antimicrobial defense substances (e.g., WRKY transcription factors and proteins encoded by the genes from the "PEN" pathway required for penetration resistance to filamentous pathogens). Thus, this work provides an inventory of candidate phosphoproteins, including putative direct MAPK substrates, for future analysis of MAPK-mediated defense control. (Proteomics data are available with the identifier PXD001252 via ProteomeXchange, http://proteomecentral.proteomexchange.org).

Keywords: MAPK substrates, defense, phosphoproteomics, metabolomics, phytoalexins, phosphorylation

\section{INTRODUCTION}

As sessile organisms, plants rely predominantly on physical barriers and adaptive mechanisms to adapt to biotic and abiotic stress conditions. During infection, pathogens can trigger PTI (pattern-triggered immunity) after recognition of conserved microbe-associated molecular patterns (MAMPs) or damageassociated molecular patterns (DAMPs), which are plant endogenous components released during tissue wounding, e.g., from herbivores or lytic enzymes released by pathogens. Binding of these MAMP/DAMP ligands to surface exposed pattern recognition receptors (PRRs) leads to activation of a variety of defense reactions to counteract the negative impact of the stresses (Boller and Felix, 2009; Knogge et al., 2009; Nicaise et al., 2009; Zipfel, 2009; Ranf et al., 2011).

The signaling network after MAMP/DAMP recognition includes ion fluxes (including a rise in cytosolic calcium concentrations), accumulation of reactive oxygen species and various phosphorylation events (Knogge et al., 2009; Liese and Romeis, 2013). Phosphorylation events appear to be key regulatory steps since treatment with kinase or phosphatase inhibitors blocks subsequent downstream signaling (Nürnberger et al., 1994; Jabs et al.,
1997; Blume et al., 2000). Clearly, one of the initial targets of such inhibitors would be the receptor-like kinases and their associated kinases/phosphatases (Monaghan and Zipfel, 2012), which are involved in the MAMP/DAMP perception. Downstream of these receptor complexes are also intracellular phosphorylation events and cascades, such as calcium-dependent protein kinases (CDPKs) (Schulz et al., 2013) and mitogen-activated protein kinase (MAPK) cascades (Pitzschke et al., 2009; Andreasson and Ellis, 2010; Tena et al., 2011; Meng and Zhang, 2013).

MAPK cascades comprise a series of at least three kinases, with the MAPK kinase kinase (MAP3K) phosphorylating a MAPK kinase (MKK) that then subsequently phosphorylates specific MAPKs at conserved threonine and tyrosine motifs. This dual phosphorylation at so-called "TXY" motifs (i.e., threonine and tyrosine separated by typically a glutamic or aspartic acid) causes a conformational change that enhances the kinase activity. The MAPK itself then phosphorylates its specific substrates to alter gene expression or modulate the activity of enzymes/proteins. At least 4 MAPKs are known to be activated after MAMP perception in Arabidopsis thaliana (Bethke et al., 2012; Eschen-Lippold et al., 2012). So far, only a few targets of these MAP kinases 
are known and most are validated only through in vitro assays (Meng and Zhang, 2013). The MAPK, MPK6, phosphorylates Nitrate Reductase 2 (NR2/NIA2) (Wang et al., 2010), and 1Aminocyclopropane-1-Carboxylate Synthase 6 (ACS6) (Liu and Zhang, 2004; Joo et al., 2008), leading to enhanced stability or activity of the enzyme and thereby to higher ethylene and NO levels, respectively. MPK4 and its substrate, MKS1, form a complex in unstressed tissues; upon phosphorylation, the MKS1 substrate is released from the complex and thought to effect transcription together with WRKY transcription factors such as WRKY33 (Andreasson et al., 2005; Qiu et al., 2008). WRKY33 is also targeted by two other MAPKs, MPK6, and MPK3, where phosphorylated WRKY33 appears to positively regulate expression of the PAD3 (Phytoalexin-Deficient 3) gene. PAD3 encodes a P450 enzyme (CYP71B15) that catalyzes the last step of the biosynthesis of camalexin, a major phytoalexin in Arabidopsis (Mao et al., 2011). Other MAPK substrates with roles in stress responses include the transcription factors ERF104 (Bethke et al., 2009), ERF6 (Meng et al., 2013) and VIP1 (Djamei et al., 2007), the universal stress proteins PHOS32/34 (Merkouropoulos et al., 2008), as well as the Tandem Zinc Finger protein 9 (TZF9) (MaldonadoBonilla et al., 2014). However, the diversity of MAPK functions in multiple cellular pathways suggests that many more MAPK substrates remain to be discovered.

Here, we studied transgenic gain-of-function Arabidopsis thaliana plants expressing a Petroselinum crispum constitutively active MKK5 protein under the control of a dexamethasone (DEX)-inducible promotor. Kinase assays following immunoprecipitation with specific MAPK antibodies have shown that transient expression in Arabidopsis of this heterologous MKK5 activated specifically two of the stress activated MAPKs, MPK3, and MPK6 but not MPK4/11 (Bethke et al., 2009). An "omics" experiment encompassing both metabolomics and proteomics was performed on the transgenic plants, which revealed a strong reprogramming of the defense metabolome and proteome. A novel phosphoproteomics procedure (Lassowskat et al., 2013) was used to enrich for phosphoproteins from leaf material, enabling us to uncover hundreds of putative phosphoproteins with altered abundance downstream of MPK3/6 activation. The presence of known MAPK substrates in this list of phosphoproteins supports the notion that novel MPK3/6 substrate candidates can be found using this strategy. Some of these candidates are regulators for the observed metabolomics reprogramming. Hence, by linking proteomics and metabolomics, this study reveals a whole range of adaptations in planta by just activating the two kinases, MPK3 and MPK6.

\section{MATERIALS AND METHODS

PLANT MATERIAL AND GROWTH

Arabidopsis thaliana (Col-0) was transformed with a constitutively-active variant of MKK5 (designated as MKK5 ${ }^{\mathrm{DD}}$ or DD) from Petroselinum crispum (Lee et al., 2004), under the control of a dexamethasone(DEX)-inducible promoter (Aoyama and Chua, 1997). As a control, the Col-0 genotype was additionally transformed with a kinase-inactive MKK5 mutant (designated as $\mathrm{MKK} 5^{\mathrm{KR}}$ or KR). These two transgenic lines are hereafter abbreviated as Col-0 DD or Col-0 KR, respectively.
For the MKK5 ${ }^{\mathrm{DD}}$ construct, transformation was also performed with the MAPK mutants, mpk3 (Miles et al., 2005) and mpk6-3 (Wang et al., 2008), the ethylene-insensitive mutants, ethyleneinsensitive2 (ein2-1) and ethylene-insensitive3 (ein3-1)/ein3-like 1 (eil1-1) double mutant (An et al., 2010), as well as the NADPH oxidase mutant (respiratory burst oxidase homologD, rbohD) (Torres et al., 2002). After selection for hygromycin resistance, putative transformants were validated for DEX-inducible expression of the myc-tagged MKK5 protein by western blot analysis. This resulted in the following independent transformants: 4 Col-0 KR, 14 Col-0 DD, 2 mpk3 DD, 4 ein2 DD and only one transgenic line for mpk6 DD, rbohD DD or ein3/eil1 DD. Stability of transgene expression was monitored by western blotting in the F2 generation and segregation of the DEX-inducible cell death phenotype (for the MKK5 ${ }^{\mathrm{DD}}$ transgenic lines) was tested in the F3 generation. After these initial characterizations, a representative line-with comparable DEX-inducible expression level of the MKK5 protein-was selected for each genotype for subsequent analysis.

Seeds were sown on soil, stratified for 2 days at $4^{\circ} \mathrm{C}$, and after transfer to individual pots, the plants were eventually maintained under short-day conditions ( $8 \mathrm{~h}$ day light, $200 \mu \mathrm{E}, 23^{\circ} \mathrm{C}$ ) for 6 weeks prior to DEX treatment (20 $\mu \mathrm{M}$ DEX in $0.0075 \%$ SILWET L-77). Three biological replicates were collected with each sample (genotype or timepoint) consisting of pooled leaf material from six different plants.

\section{WESTERN BLOT}

Protein extraction and immunoblot with anti-pTEpY $(\alpha-$ phospho-p44/42-ERK; CST, www.cellsignal.com) to detect activated forms of the MAPKs or c-myc epitope to detect the MKK5 proteins were performed as described (Ranf et al., 2011).

\section{PROTEOMICS ANALYSIS}

\section{Protein extraction and phosphoprotein enrichment}

Details to total protein and phosphoprotein enrichment via the Prefractionation-assisted Phosphoprotein Enrichment (PAPE) procedure can be found in Lassowskat et al. (2013). PAPE is a two-step fractionation with $40 \%$ ammonium sulfate precipitation that apparently enriches phosphoproteins (Lassowskat et al., 2013), followed by a metal oxide affinity chromatography (MOAC) step (Wolschin and Weckwerth, 2005). Typically, $25 \mathrm{~g}$ of frozen leaf material were ground to a fine powder in liquid nitrogen and extracted by vigorous shaking $\left(20 \mathrm{~min}, 4^{\circ} \mathrm{C}\right)$ with three volumes (w/v) of extraction buffer (100 mM HEPES-KOH, $\mathrm{pH} 7.5,5 \%$ glycerol, $5 \mathrm{mM}$ EDTA, $0.1 \%$ (v/v) B-mercaptoethanol, $1 \%(\mathrm{v} / \mathrm{v})$ protease and phosphatase-inhibitor II and III mix from Sigma-Aldrich). After centrifugation $\left(3220 \times \mathrm{g}, 4^{\circ} \mathrm{C}, 15 \mathrm{~min}\right)$ and filtering through a 0.45 micron Rotilabo ${ }^{\circledR}$ cellulose mixed ester (CME) filter (Roth, http://www.carlroth.com), the supernatant was either used for $40 \%$ ammonium sulfate precipitation (first step of PAPE as described; Lassowskat et al., 2013) or mixed with Tris-EDTA-buffered phenol (for the total protein fraction). The lower organic phase after centrifugation $\left(3220 \times \mathrm{g}, 4^{\circ} \mathrm{C}, 15 \mathrm{~min}\right)$ was re-extracted (with an equal volume of $100 \mathrm{mM}$ Tris- $\mathrm{HCl}$, pH8.4, $20 \mathrm{mM} \mathrm{KCl,} 10 \mathrm{mM}$ EDTA, 0.4\% ß-mercaptoethanol). Proteins in the phenol phase were precipitated overnight $\left(-20^{\circ} \mathrm{C}\right)$ 
with five volumes of cold precipitation solution (methanol containing $100 \mathrm{mM}$ ammonium acetate). The protein pellet $(3220 \times$ $\mathrm{g}, 4^{\circ} \mathrm{C}, 15 \mathrm{~min}$ ) was rinsed with precipitation solution and twice with a cold mixture $\left(-20^{\circ} \mathrm{C}\right)$ of $80 \%(\mathrm{v} / \mathrm{v})$ acetone $/ 20 \%$ Tris- $\mathrm{HCl}$, $\mathrm{pH}$ 7.5. After 5 min drying, the pellet was resuspended in shotgun buffer ( $50 \mathrm{mM}$ Tris- $\mathrm{HCl}, \mathrm{pH} 8.5,8 \mathrm{M}$ urea) to obtain the total protein extract.

For the second step in the PAPE procedure, strict adherence to the protein:metal matrix ratio is essential for the MOAC step. Typically, a $1.5 \mathrm{ml}$ protein sample $(0.5 \mathrm{mg} / \mathrm{ml})$ was incubated with $40 \mathrm{mg}$ of $\mathrm{Al}\left(\mathrm{OH}_{3}\right)$ (Sigma-Aldrich) that had been washed and equilibrated in MOAC incubation buffer. After $30 \mathrm{~min}\left(4^{\circ} \mathrm{C}\right)$ rotation, the metal oxide was precipitated $(18514 \times \mathrm{g}, 2 \mathrm{~min})$ and rinsed four times with incubation buffer. The proteins were eluted twice $(800$ and $400 \mu \mathrm{l})$ with tetrapotassium pyrophosphate (TKPP) buffer ( $8 \mathrm{M}$ urea, $100 \mathrm{mM}$ TKPP, $\mathrm{pH} 9.0$ ) for $45 \mathrm{~min}$ at room temperature. The pooled eluates were centrifuged twice $\left(18514 \times \mathrm{g}, 2 \mathrm{~min}, 15^{\circ} \mathrm{C}\right)$, to pellet any remaining matrix, and concentrated with centricon filter devices $(3 \mathrm{kDa}$ cut-off; Millipore, http://www.merckmillipore.com). Proteins were precipitated with a 2D-CleanUp kit (GE Healthcare, http://www. gelifesciences.com), according to the manufacturer's instructions, and solubilized in shotgun buffer.

\section{In-solution digestion}

Protein concentration was determined by 2-D Quant Kit (GE Healthcare, www.gelifesciences.com) and the proteins were reduced (100 $\mathrm{mM}$ DTT, $100 \mathrm{mM}$ Tris- $\mathrm{HCl}, \mathrm{pH} 7.8$, for $1 \mathrm{~h}$ ) and alkylated (200 mM iodoacetamide, $100 \mathrm{mM}$ Tris- $\mathrm{HCl}, \mathrm{pH} 7.8$, for $1 \mathrm{~h}$ ). The solution was diluted to an end concentration of $0.5 \mathrm{M}$ urea with $50 \mathrm{mM} \mathrm{NH}_{4} \mathrm{HCO}_{3}(\mathrm{pH} 8)$ and digested overnight with sequencing grade trypsin (Promega, www.promega.com) at a ratio of $1: 50$ at $37^{\circ} \mathrm{C}$. Peptides (max. $200 \mu \mathrm{L}$ ) were desalted on C18 columns (for up to $30 \mu$ g peptide samples, PepClean ${ }^{\mathrm{TM}}$, Thermo Scientific, www.piercenet.com; or for up to $1 \mathrm{mg}$ peptide samples, C18 Protea Spin Tips, Protea Biosciences, https:// proteabio.com/) according to the supplier's instructions and reconstituted in $5 \% \mathrm{ACN}, 0.1 \%$ TFA.

\section{Mass spectrometry (proteomics)}

Tryptic digests were analyzed with an LC-MS system consisting of a nano-LC (Easy-nLC II, Thermo Scientific, www.proxeon.com) coupled to a hybrid-FT-mass spectrometer (LTQ Orbitrap Velos Pro, Thermo Scientific, www.thermoscientific.com). Peptide separations were performed on a C18 column (EASY column; $10 \mathrm{~cm}$, ID $75 \mu \mathrm{m}$, particle diameter $3 \mu \mathrm{m}$ ) at a flow rate of $300 \mathrm{~nL} / \mathrm{min}$ and a linear gradient of 5-40\% B in $150 \mathrm{~min}$ for total protein measurements and $300 \mathrm{~min}$ for measurement of the phosphoproteinenriched fractions (A: $0.1 \%$ formic acid in water, B: $0.1 \%$ formic acid in $\mathrm{ACN})$. A voltage of $+1.9 \mathrm{kV}$ was applied to electrospray peptide ions. A capillary temperature of $275^{\circ} \mathrm{C}$ for peptide transfer and a lock mass of $445.120024 \mathrm{~m} / \mathrm{z}$ were used. Precursor mass scanning was performed from 400 to $1850 \mathrm{~m} / \mathrm{z}$ in the Orbitrap with a resolution of 30,000 and the 20 most intense precursor ions were selected for subsequent CID fragmentation in the linear trap quadrupole mass analyzer (LTQ). Singly charged ions were rejected from fragmentation. Dynamic exclusion was enabled
(Repeat Count: 1, Repeat Duration: 20 s, Exclusion List Size: 500, Exclusion Duration: 30 s).

\section{Spectral data analysis}

Each sample (genotypes/treatments (i.e., transgene)/timepoints) is based on three biological replicates, which are each measured twice (resulting in six values per sample). The resulting MS raw data were analyzed with the Progenesis LC-MS software (Nonlinear Dynamics Limited, www.nonlinear.com). After alignment and feature detection, normalization was applied automatically to all features as recommended by the Progenesis LC-MS software manual. Resulting features were filtered for an ANOVA $p$-value of $<0.05$ and fold-change of $>2.0$ (between the $\mathrm{MKK} 5^{\mathrm{DD}}$ and $\mathrm{MKK} 5^{\mathrm{KR}}$ plants). Available MS spectra with rank 10 and better were searched against an A. thaliana protein database based on TAIR10 (www.arabidopsis.org) using an in-house Mascot server (with the following parameters: precursor mass tolerance: $7 \mathrm{ppm}$, fragment mass tolerance: $0.8 \mathrm{Da}$, missed cleavages: 2). Carbamidomethylation of cysteine was set as a static modification. Variable modifications were oxidation (M), acetylation of protein N-terminus, deamidation (NQ), and phosphorylation (STY). Peptides with Mascot scores less than 20 were rejected. Protein grouping and quantitation from nonconflicting peptides was enabled. Normalized abundance relative to maximum (Tables S04-S09) was calculated based on normalized abundance from Progenesis LC-MS. Maximum value of each protein (from 0 to $24 \mathrm{~h}$ samples) was set as one, with all other values being relative to this. Phosphopeptides were identified with the Proteome Discoverer 1.4 software, which includes the phosphoRS 2.0 algorithm (Thermo Fisher Scientific) for phospho-site mapping. A false discovery rate (FDR) was calculated by searching a "decoy" database containing all the target database sequences in reverse order. Peptide-spectrum match (PSM) was set at a $q<0.05$ (i.e., a corrected significance threshold employing the Benjamini-Hochberg FDR procedure to control for a family-wise error rate).

\section{Targeted proteomics analysis}

The LC method with $300 \mathrm{~min}$ gradient as well as the MS acquisition method was identical to point Mass spectrometry (proteomics) with the following changes. A global parent mass list was used (Table S20) and dynamic exclusion was disabled. Parent masses were extracted from previous measurements of phosphopeptides. Spectral data analysis was performed with Proteome Discoverer 1.4 according to parameters described in point Spectral data analysis. The number of PSMs was calculated as a sum from measurements of the four samples (collected 4, 5, 7, $8 \mathrm{~h}$ after DEX treatment).

\section{METABOLOMICS ANALYSIS}

\section{Metabolite extraction}

Frozen leaf material was homogenized in liquid nitrogen and weighed ( $50 \pm 2 \mathrm{mg}$ ) into a pre-cooled 2-ml polypropylene tube. After addition of $80 \%$ methanol $(200 \mu \mathrm{L})$, pre-cooled at $-80^{\circ} \mathrm{C}$ and spiked with indole-3-acetyl-L-valine $(1 \mu \mathrm{M})$, o-anisic acid $(1 \mu \mathrm{M})$, and kinetin $(2 \mu \mathrm{M})$ as internal standards the sample was allowed to reach room temperature under vigorous shaking 
within $5 \mathrm{~min}$. The resulting mixture was sonicated $\left(10 \mathrm{~min}, 20^{\circ} \mathrm{C}\right)$ and centrifuged at $11,200 \times \mathrm{g}\left(10 \mathrm{~min}, 16^{\circ} \mathrm{C}\right)$. The sediment was re-extracted with $80 \%$ methanol $(200 \mu \mathrm{L})$. Both supernatants were combined and evaporated to dryness under reduced pressure at $40^{\circ} \mathrm{C}$ using a vacuum centrifuge. The remaining residue was thoroughly reconstituted in $30 \%$ methanol $(150 \mu \mathrm{L})$, sonicated $\left(15 \mathrm{~min}, 20^{\circ} \mathrm{C}\right)$ and centrifuged at $11,200 \times \mathrm{g}(10 \mathrm{~min}$, $\left.16^{\circ} \mathrm{C}\right)$. An $80-\mu \mathrm{l}$ aliquot of the supernatant was transferred into an autosampler vial and subjected to LC/MS analysis in both positive and negative ion mode. For quantification of abundant metabolites, a $30-\mu \mathrm{L}$ aliquot of the supernatant was diluted with $120 \mu \mathrm{L}$ methanol/water, 3/7 (v/v) and subjected to LC-MS analysis in negative ion mode.

\section{Mass spectrometry (metabolomics)}

Leaf extracts were analyzed with an LC-MS system consisting of an UPLC (Acquity, Waters, www.waters.com) coupled to a hybrid quadrupole time-of-flight mass spectrometer (micrOTOF-Q I, Bruker Daltonics, www.bruker.com) equipped with an Apollo II electrospray ion source. Chromatographic separations were performed at $40^{\circ} \mathrm{C}$ on a HSS T3 column $(100 \times 1.0 \mathrm{~mm}$, particle size $1.8 \mu \mathrm{m}$, Waters) applying the following binary gradient at a flow rate of $150 \mu \mathrm{L} \mathrm{min}^{-1}: 0-1 \mathrm{~min}$, isocratic $95 \% \mathrm{~A}(0.1 \%$ formic acid in water $), 5 \% \mathrm{~B}$ (0.1\% formic acid in acetonitrile); $1-10 \mathrm{~min}$, linear from 5 to $60 \% \mathrm{~B} ; 10-12 \mathrm{~min}$, isocratic $95 \% \mathrm{~B} ; 12-14 \mathrm{~min}$, isocratic $5 \% \mathrm{~B}$. The injection volume was $2.6 \mu \mathrm{L}$ (full loop injection). Eluted compounds were detected from $m / z 100-1000$ in positive (negative) ion mode using the following instrument settings: nebulizer gas, nitrogen, $1.6 \mathrm{bar}$; dry gas, nitrogen, $6 \mathrm{~L} / \mathrm{min}, 190^{\circ} \mathrm{C}$; capillary, $-5000 \mathrm{~V}(+4000 \mathrm{~V})$; end plate offset, $-500 \mathrm{~V}$; funnel $1 \mathrm{RF}, 200 \mathrm{Vpp}$; funnel $2 \mathrm{RF}, 200 \mathrm{Vpp}$; in-source CID energy, $0 \mathrm{~V}$; hexapole RF, $100 \mathrm{Vpp}$; quadrupole ion energy, $3 \mathrm{eV}$; collision gas, argon; collision energy, $3 \mathrm{eV}(10 \mathrm{eV})$; collision RF 200/400 Vpp (timing 50/50); transfer time, $70 \mu \mathrm{s}$; pre pulse storage, $5 \mu \mathrm{s}$; pulser frequency, $10 \mathrm{kHz}$; spectra rate, $3 \mathrm{~Hz}$. Mass spectra were acquired in centroid mode. Calibration of the $m / z$ scale was performed for individual raw data files on lithium formate cluster ions obtained by automatic infusion of $20 \mu \mathrm{L} 10 \mathrm{mM}$ lithium hydroxide in isopropanol/water/formic acid, 49.9/49.9/0.2 (v/v/v) at a gradient time of 12 min using a diverter valve. For aquisition of collisioninduced dissociation mass spectra appropriate precursor ions were selected using an isolation width of $\pm 4 \mathrm{~m} / \mathrm{z}$ and fragmented inside the collision cell applying collision energies in the range of 10-40 eV. Product ions were detected using the same parameter settings as described above, except for spectra rate $(1.5 \mathrm{~Hz})$ and collision RF [100/200 Vpp (timing 50/50)].

\section{Non-targeted data analysis and metabolite annotation}

A total of 48 undiluted methanolic leaf extracts [genotype $\times$ treatment time $\times$ replicate $=($ Col-0 DD, Col-0 KR $) \times(0,4,8,12,16$, $20,24,36 \mathrm{~h}) \times 3$ ] were analyzed by UPLC/ESI-QTOFMS in positive and negative ion mode resulting in a total of 96 raw data files. To judge analytical quality and estimate alignment parameters, retention times and responses of spiked internal standards were determined prior to non-targeted data analysis (retention time deviation $\approx \pm 2.5 \mathrm{~s}$, coefficient of variation of internal standard responses $\leq 11 \%$ ). Raw data files were converted into mzData format using CompassXPort (Bruker Daltonics) and processed for each ion mode in two batches of 48 raw data files using the R package XCMS (Smith et al., 2006). Raw data files were arranged in 16 sample classes according to genotype and treatment time. Feature detection was performed using the centWave algorithm $[$ sntresh $=3$, prefilter $=(3,100), \mathrm{ppm}=25$, peak width $=(5,12)]$. Alignment was accomplished by consecutively applying the XCMS functions group.density ( $\operatorname{minfrac}=1$, bw $=2$ and mzwid $=0.05)$, retcor $(\operatorname{span}=1$, missing $=0$, extra $=0)$, and group.density $(\operatorname{minfrac}=1$, bw $=1$ and mzwid $=0.03)$. Missing feature intensities were filled in using the XCMS function fillPeaks. The resulting intensity matrix (feature $\times$ sample) was log2-transformed and subjected to Two-Way ANOVA. Features with a significant genotype-treatment time interaction $(p<0.01)$ were filtered by intensity (maximum intensity $\geq 2^{14}$ counts). The resulting feature sets (positive and negative ion mode) were used for reconstruction and annotation of the underlying compound mass spectra which was aided by the R package CAMERA (Kuhl et al., 2012) and manual analyses using DataAnalysis 4.0 (Bruker Daltonics). High-resolution CID mass spectra of annotated quasi-molecular ions were acquired. Elemental compositions were calculated on accurate $\mathrm{m} / \mathrm{z}$ of quasi-molecular ions within an error range of $10 \mathrm{ppm}$ and filtered by relative isotope abundance, number of double bond equivalents and electron parity using the SmartFormula algorithm implemented in DataAnalysis 4.0. Putative elemental compositions were further checked for consistency by analysis of elemental compositions of fragment ions. Compounds were then putatively annotated by interpretation of characteristic fragment ions and neutral losses. Annotation of a total of 23 metabolites could be verified by analysis of reference compounds. Analytical data and mass spectral characterization is given in Tables S1, S2. Finally, all annotated compounds were manually quantified by integration of extracted ion chromatograms using QuantAnalysis 2.0 (Bruker Daltonics) and retention times/quantifier ions from Table S19.

\section{RESULTS TRANSGENIC EXPRESSION OF A CONSTITUTIVELY ACTIVE MKK5 (MKK5 ${ }^{\mathrm{DD}}$ ) LEADS TO ACTIVATION OF MPK3 AND MPK6}

Substitution of two serines by "phosphomimicking" aspartic acid (D) in the kinase activation loop of the parsley MAPK kinase 5 (MKK5) generates a constitutively active kinase (abbreviated as $\mathrm{MKK} 5^{\mathrm{DD}}$ ) that can phosphorylate and activate downstream MAPKs (Lee et al., 2004). Transgenic Arabidopsis thaliana (Col-0) plants were created with a dexamethasone (DEX)-inducible expression of this heterologous MKK5 ${ }^{\mathrm{DD}}$ (hereafter named Col-0 DD) (see material and methods for generation and validation of the transgenic plants). Two immunoreactive bands representing phosphorylated MAPKs are detected in plant extracts of such transgenic plants after 4-6h of DEX treatment (Figure 1A), which is not seen in corresponding control plants (Col-0 KR) that express the kinase-inactive version of MKK5 $\left(\mathrm{MKK} 5{ }^{\mathrm{KR}}\right)$. Previous experiments using MAPK-specific antibodies have defined these two bands to be MPK3 and MPK6 (Bethke et al., 2009) and this is now further confirmed by the loss of one of the MAPK bands in the respective $m p k 3$ or $m p k 6$ mutant background (see Figure S1). 


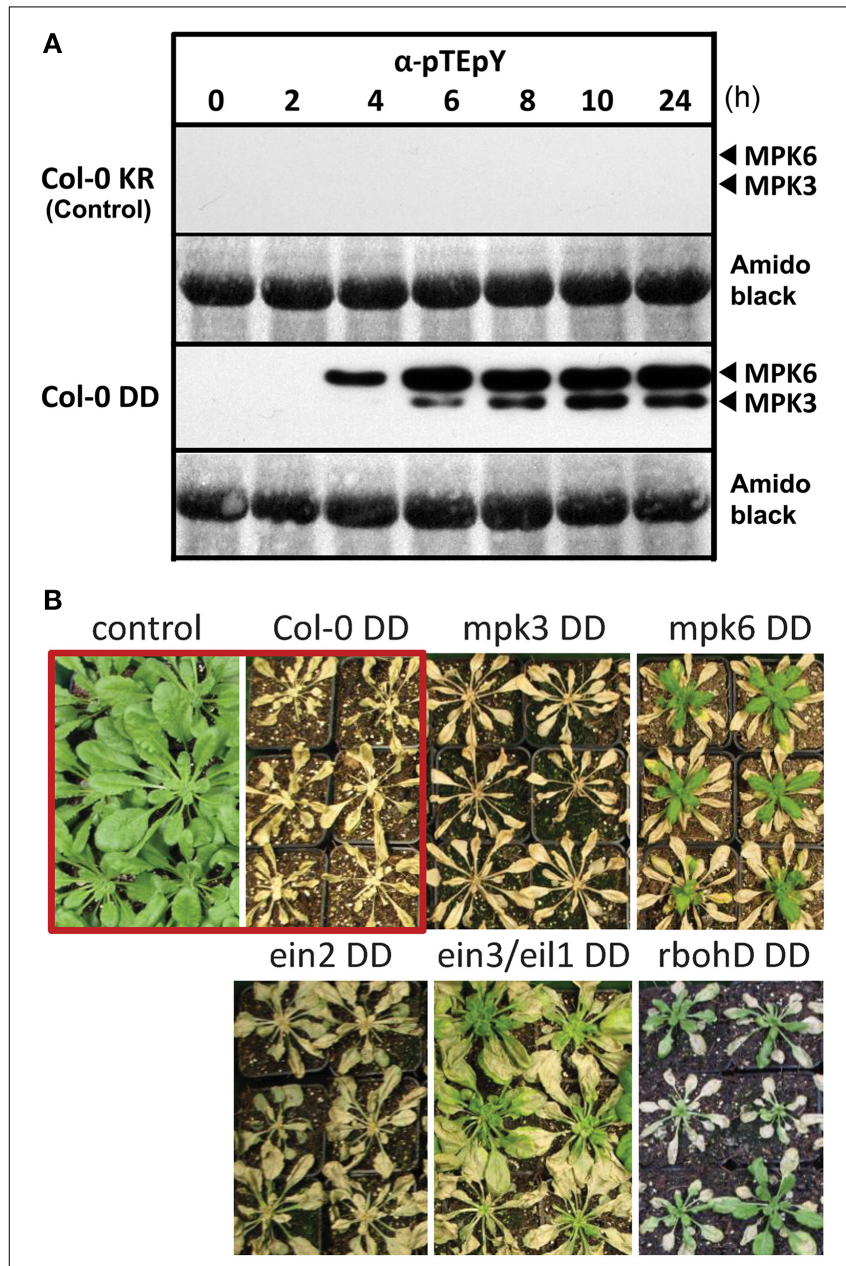

FIGURE 1 | Activation of the MAP kinases, MPK 3 and MPK6, by DEX-inducible expression of a constitutively active MAPK kinase, MKK5. (A) Six week old plants were sprayed with $20 \mu$ M DEX to induce expression of a constitutively active MAPK kinase $5, M K K 5^{D D}$, in Col-0 ecotype background (abbreviated as Col-0 DD). Leaves were harvested at the indicated time points after DEX treatment and used for immunodetection of phosphorylated MAPKs ( $\alpha-p T E p Y)$. The expected positions of MPK3/6 are marked. Col-0 KR designates the corresponding control plants expressing a kinase-inactive version of MKK5. (B) Prolonged MPK3/6 activation, after DEX treatment, lead to death of the plants-visible as tissue collapse after $24 \mathrm{~h}$. (Note that for some lines, new leaves emerged from the central meristem some days after the DEX treatment). Photos of the plants were taken 2 week after DEX treatment. The core experiment (within red box) compares the Col-0 KR (control) with Col-0 DD (active $M K K 5^{\mathrm{DD}}$ ) lines. Additional transgenic lines expressing the $\mathrm{MKK} 5^{\mathrm{DD}}$ in the indicated mutant background are also included (For detection of phosphorylated MAPKs for these lines, please see Figure S1).

A prolonged activation of MPK3 and/or MPK6 leads to tissue collapse within $24-48 \mathrm{~h}$, and to complete death after 4-7 days post DEX treatment (Figure 1B). Similar experimental set-up with the tobacco MKK5 ortholog has pointed to subsequent downstream ethylene and reactive oxygen species (ROS) production (Ren et al., 2002; Liu and Zhang, 2004). To evaluate the influence of such downstream signaling molecules and also the individual contribution of MPK3 or MPK6, the MKK5 $5^{\mathrm{DD}}$ construct was also transformed in $m p k 3, m p k 6$, respiratory burst oxidase homologD (rbohD), ethylene-insensitive2 (ein2) mutants, as well as the ethylene-insensitive3/ein3-like1 (ein3/eil1) double mutant. DEX-induced death of the plants was seen in all these genetic backgrounds. However, new leaves developed from the central meristem for the mpk6, rbohD, and ein3/eill double mutants 7-14 days later (Figure 1B); thus pointing to roles of the corresponding genes in the cell death phenotype. Taken together, the transgenic system described here simulates MPK3/6 activation after pathogen attack without any direct exposure to pathogens or any pathogen-derived molecules (e.g., MAMPs).

\section{METABOLIC OUTCOME OF SIMULATED PATHOGEN DEFENSE}

To investigate the impact of artificial MPK3/6 activation on plant defense metabolism without any complications from pathogens, we performed non-targeted metabolite profiling for semi-polar secondary metabolites. Samples were collected 0, 4, 8, 12, 16, 20, 24 and $36 \mathrm{~h}$ after DEX treatment in three independent experiments. These timepoints were selected to cover the initial time period where the MPK3/6 activation are just detectable $(\sim 4-6 \mathrm{~h})$ (Figure 1A) until (and after) the initiation of tissue collapse (24$48 \mathrm{~h}$ ). Note that at $36 \mathrm{~h}$, most tissues from the MKK5 ${ }^{\mathrm{DD}}$ plants are partially collapsed and flaccid, but not completely dead (i.e., brown and dry, see Figure S1B). A total of 12,913 and 10,731 molecular features were extracted from LC-MS data acquired in the positive and negative ion mode, respectively. After setting an intensity filter cutoff ( $>2^{14}$ counts) and Two-Way ANOVA $(p<0.01), 853$ and 724 differential features remained, which were further subjected to metabolite annotation (for analytical data see Tables S1, S2). In combination with known leaf metabolites this resulted in a total of 113 compounds, including 24 unidentified substances (Figure 2A). Many known defenserelated compounds accumulated to high levels (see Figure 2A for compound classification and Table S1 for a complete list). The most prominent and numerous are a variety of tryptophan (Trp)-derived defense metabolites (camalexin and indole glucosinolate derivatives) as well as Trp-derived indole-3-carboxylic acid derivatives (Figure 2B and Figure S2A).

Various indole glucosinolate (GLS) derivatives, initially described to be associated with herbivore resistance (Barth and Jander, 2006; Winde and Wittstock, 2011; Stauber et al., 2012; Schweizer et al., 2013) and subsequently to penetration resistance by filamentous pathogens (Bednarek et al., 2009; Clay et al., 2009), were found to accumulate rapidly. In particular, accumulation of indole-3-ylmethylamine $\left(\mathrm{I}_{3} \mathrm{MNH}_{2}\right)$ and its 4-methoxy derivative $\left(4 \mathrm{MeOI} 3 \mathrm{MNH}_{2}\right)$ were observed already after $4-6 \mathrm{~h}$ DEX treatment (Figure 2C). This is almost concomitant with the MPK3/6 activation (cf. Figure 1A). Similarly, levels of raphanusamic acid and $4 \mathrm{MeOI} 3 \mathrm{M}$ GLS also start to rise within $6 \mathrm{~h}$. By contrast, the precursor metabolite, indole-3-ylmethyl (I3M) GLS, is reduced $\sim 8 \mathrm{~h}$ after DEX treatment, possibly as a consequence of heightened flux into the downstream metabolites. I3M GLS is synthesized from indole-3-acetaldoxime (IAOx), which can additionally be converted into indole-3-acetonitrile (IAN) by the cytochrome P450 enzyme CYP71A13 (Nafisi et al., 2007) (Figure 2B). IAN can further be conjugated to glutathione, and converted into the phytoalexin, camalexin. Indeed, camalexin, and its precursors 


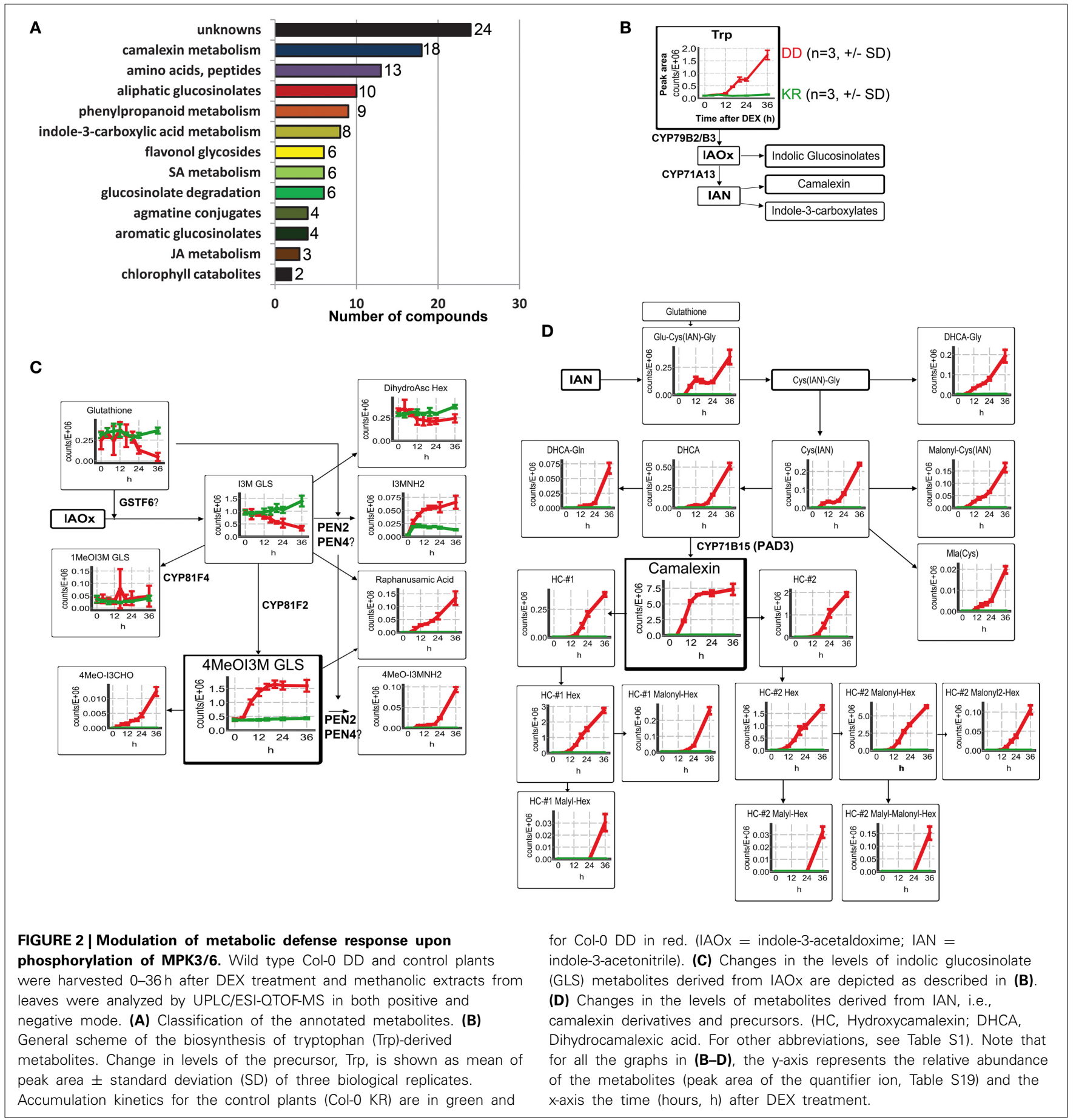

rapidly accumulated $6 \mathrm{~h}$ after DEX treatment, with camalexin abundance reaching a plateau phase within $12 \mathrm{~h}$, which is followed by an increase of various hydroxy-camalexins (HC) and their conjugates ( $\sim 12-24 \mathrm{~h}$ ) (Figure 2D). Since these compounds can already be detected prior to any obvious macroscopic tissue damage, their accumulation is unlikely to be caused by tissue wounding or cell death. Hence, artificial activation of MPK $3 / 6$ is sufficient to cause massive accumulation of the major Arabidopsis antimicrobial metabolites.
Induced metabolite accumulation was also seen for some anabolites and catabolites of Met-derived GLS, as well as many derivatives of indole-3-carboxylic acid, agmatine conjugates and various aromatic amino acids. By contrast, little to no changes could be seen for flavonol glycosides and hydroxycinnamate esters (Figures S2A-F). However, some of the hydroxycinnamic acids were probably channeled into the accumulating feruloyl or coumaroyl agmatines (cf. Figure S2C). In addition to the previously described increase of ethylene production after MPK3/6 
activation (Liu and Zhang, 2004), precursors and conjugates of defense-related phytohormones such as salicylic acid (SA) and jasmonic acid (JA) were also detected (Figures S2G,H). In agreement with the observed tissue collapse and cell death (Figure 1A), two non-fluorescent chlorophyll catabolites, NCC1 and NCC4, start to appear $12-14 \mathrm{~h}$ and are detected at high levels $36 \mathrm{~h}$ after DEX treatment (Figure S2I).

To check which of the two MAPKs or whether downstream signaling through ethylene or ROS contributes to the accumulation of these metabolites, we compared in various mutant backgrounds the levels of three selected defense-related metabolites known to have antimicrobial activities against pathogens/microbes $24 \mathrm{~h}$ after DEX treatment. MPK6 is clearly required for the DEX-induced accumulation of camalexin,
4MeOI3M GLS and acetylagmatine (Figures 3A-C). On the other hand, MPK3 — while not absolutely essential—contributes to reaching maximum acetylagmatine levels but appears to be dispensable for camalexin and 4MeOI3M GLS accumulation. Camalexin production is independent of ethylene signaling but required ROS generated by the NADPH oxidase, RBOHD (Figure 3A). By contrast, acetylagmatine accumulation required both ethylene and ROS signaling (Figure 3C); while 4MeOI3M GLS appears to require neither ethylene nor ROS from RBOHD (Figure 2B). On the other hand, no statistically significant changes between the tested mutants was observed (Figure 3D) for the $4 \mathrm{MeOI} 3 \mathrm{M}$ GLS precursor, I3M GLS, which did not show strong DEX-induced accumulation (cf. Figure 2C). In summary, the DEX-induced accumulation of three major antimicrobial

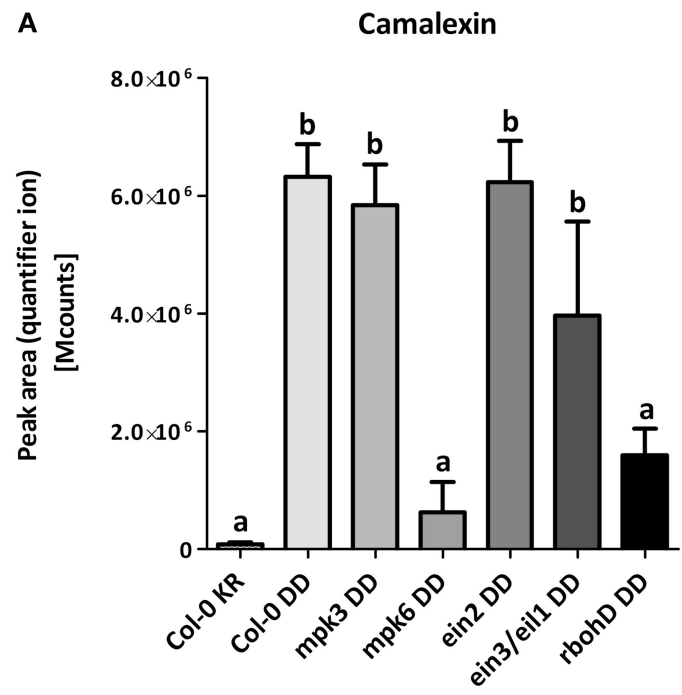

C

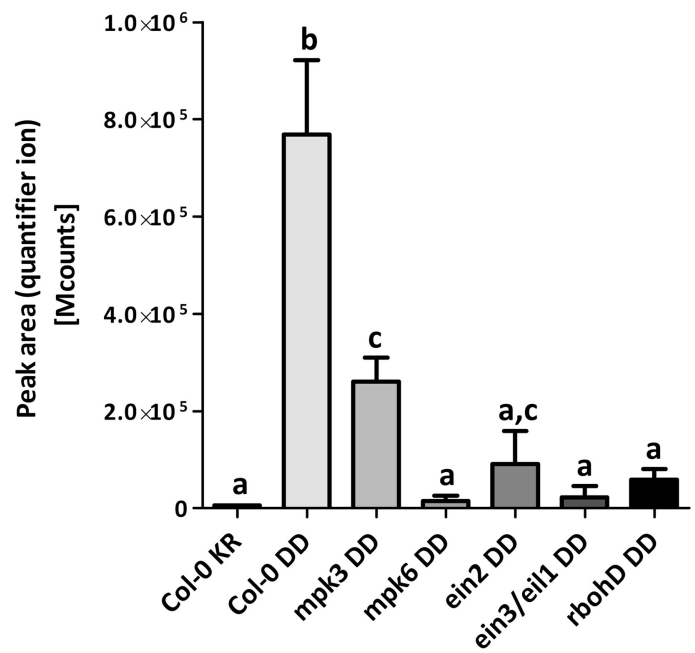

FIGURE 3 | Mutant background influences metabolome modulation. Samples were taken $24 \mathrm{~h}$ after DEX treatment in three independent experiments. The peak areas of the quantifier ion (Table S19) for camalexin (A), 4MeOI3M-GLS (B), acetylagmatine (C) and I3M-GLS (D) were determined for relative quantification. Significance of changes was

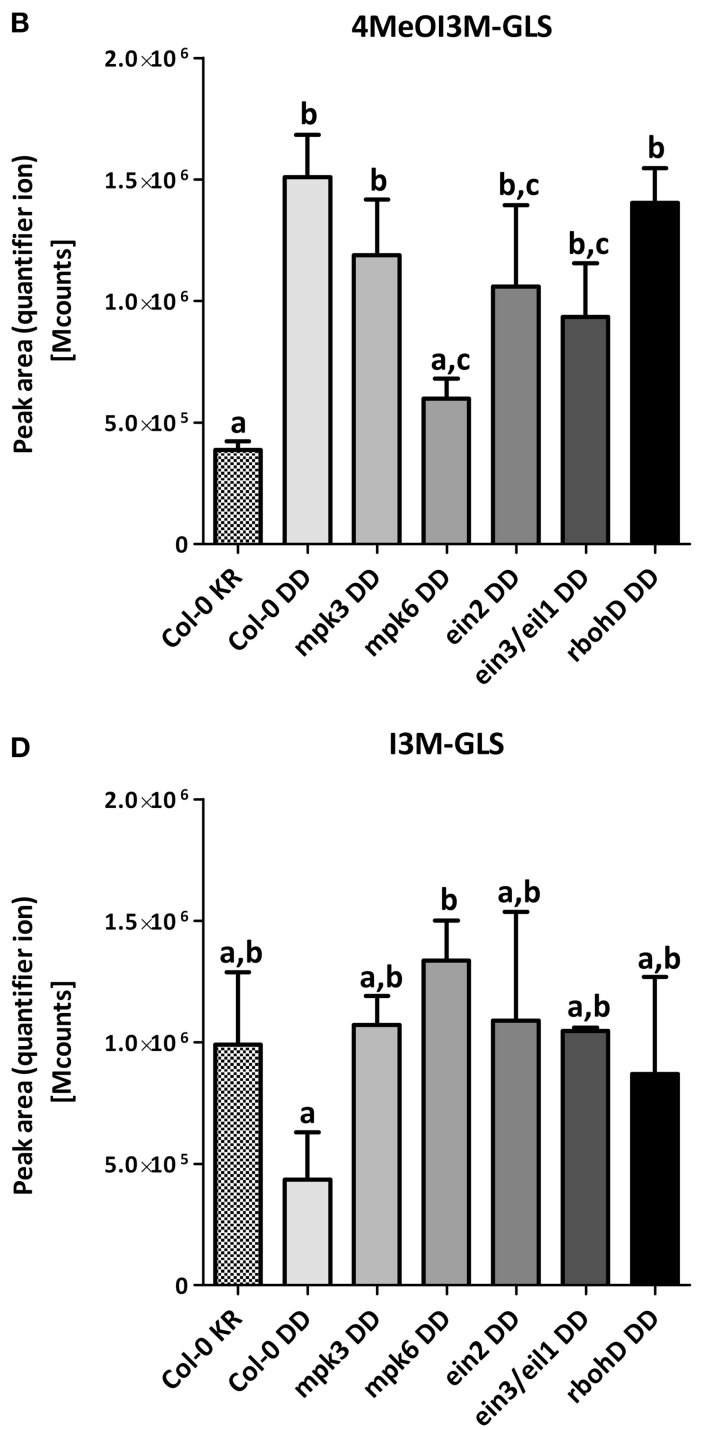

calculated by One-Way ANOVA with Tukey's multiple comparison post-test, where the different alphabets denote statistically distinct groups. Note that in $\mathrm{B}$, the levels of $4 \mathrm{MeO} 3 \mathrm{M}-\mathrm{GLS}$ in the mpk6, ein2 or ein3/eil1 mutants are not distinguishable and therefore additionally marked with the letter $c$. 
metabolites requires MPK6 activation but has differential requirements for downstream ethylene and ROS signaling to achieve similar metabolite levels as the wild-type plants.

The findings above indicate that MPK3/6 activation, without any direct exposure to pathogens or pathogen-derived MAMPs, is sufficient to trigger the accumulation of multiple antimicrobial and stress-related metabolites. This metabolic reprogramming must be regulated through the actions of MPK3/6 on their substrates as well as a change in the overall proteome. It therefore pinpoints the importance of MAPKs in activating defense reactions to counteract invading pathogens or pests.

\section{CHANGES IN THE TOTAL PROTEOME FOLLOWING MPK3 AND MPK6 ACTIVATION}

To elucidate the defense metabolism reprogramming by MPK3/6 activation, we performed a global proteome analysis of leaf material collected at $0,2,4,6,8,10,12$, and $24 \mathrm{~h}$ after DEX spraying of the transgenic $\mathrm{MKK} 5^{\mathrm{DD}}$ plants compared to the MKK5 ${ }^{\mathrm{KR}}$ control plants. The time points were selected to cover the period where the rise in activated MAPKs $(\sim 4 \mathrm{~h}$, Figure S1) and the rise in metabolites levels (typically starting at $6-12 \mathrm{~h}$ after DEX, c.f. Figure 2 and Figure S2) were detectable. Due to low protein yield, the $36 \mathrm{~h}$ timepoint was excluded. Triplicate samples (with each sample consisting of three plants pooled together) were collected for each genotype and time point. Two micrograms of extracted protein were digested with trypsin and separated with a 150-min LC gradient for LC-MS measurements. Quantitation of protein changes was performed with the Progenesis LC-MS software package and compared within each genotype. The heat map in Figure 4A illustrates the $\sim 700$ proteins with altered abundance ( $>2$-fold, ANOVA, $p<0.05$, for at least one of the measured time points) for the Col-0 genotype. Abundances for 480 and 145 proteins were gradually up- or down-regulated, respectively, during the $24 \mathrm{~h}$ period, while another group of 72 proteins showed fluctuating up- and down-regulation in an irregular manner (Figure 4A, Table S4). By contrast, the Col-0 KR plants had considerably less number of proteins with altered abundance (Table S3). With exception of the mpk3 mutant with its list of 789 proteins, all other tested mutants had fewer proteins with altered abundance (i.e., 267-501, Figure 4B, Tables S5-S9). Since the total number of detected proteins is comparable between genotypes (i.e., $\sim 2000$ in all cases, Figure 4B), the reduced number of proteins showing altered abundance in some mutants is not due to overall lower protein detection in a particular sample. Rather, it suggests that the changes in protein abundance are dependent on ethylene and ROS signaling. For $m p k 6$, the reduced number of protein changes suggests an important contribution of MPK6 but it may also be in part through diminished ethylene biosynthesis since MPK6 is a major MAPK for stabilizing ACC synthase levels through phosphorylation (Liu and Zhang, 2004; Joo et al., 2008).

Even though MPK3 and MPK6 are often mooted to have overlapping redundant functions, the results above point to a stronger role of MPK6 in mediating the observed global proteome changes. However, it is possible that the heterologous MKK5 used here may have some preference for MPK6 over MPK3 in vivo. Although in vitro kinase assays showed that recombinant parsley $\mathrm{MKK} 5^{\mathrm{DD}}$ can phosphorylate the Arabidopsis

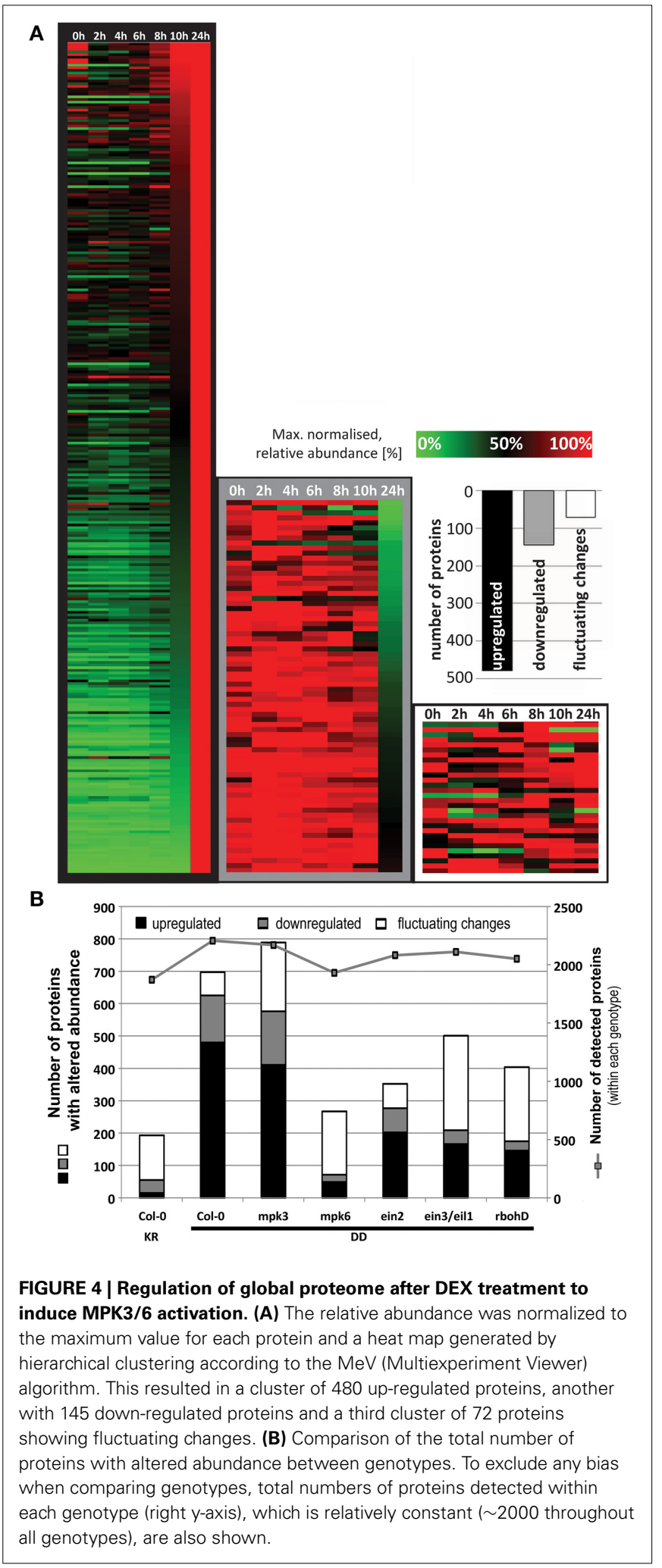

MPK3 and MPK6 equally well (not shown), the MPK3 phosphorylation by $M K K 5^{D D}$ in the mpk6 background is indeed weaker and transient (Figure S1). Hence, the profile based on the number of proteins up- or down-regulated is, as 
expected, very similar between Col-0 and the mpk3 mutant (c.f. Tables S4, S5). A "STRINGS interactome network" of the up-regulated proteins, which functionally clusters known and predicted protein-protein interactions (Franceschini et al., 2013), also revealed mostly similarities between Col-0 and $m p k 3$ (Figure 5). In agreement to the observed metabolome changes (Figure 2), up-regulated proteins with functions in primary metabolism, Trp biosynthesis, and glucosinolate metabolism, as well as gluthathione-S-transferases are prominently clustered. In addition, MAPK components, pathogenesis-related proteins and components of peptidase/proteasome degradation pathways are also pronounced. However, a cluster consisting of proteins involved in protein translation is completely missing in the $m p k 3$ mutant (Figure 5); amongst these are a number of ribosomal proteins (highlighted in yellow in Table S4). Thus, despite the apparent weaker MPK3 activation (as compared to MPK6) in the transgenic system, this unforeseen difference implies that MPK3 activation has a regulatory function on elements of the protein translational machinery, which is not replaceable by MPK6. Hence, besides their overlapping functions, MPK3 and MPK6 have distinct cellular targets.

\section{IDENTIFICATION OF PUTATIVE IN VIVO MAPK SUBSTRATES}

The findings above show that a simulated MPK3/6 activation reprograms the overall proteome and (defense) metabolome. From the rapid timing of the metabolic changes (Figure 2), one may postulate that a direct action of the activated MAPKs on their substrates is likely to be responsible for most of the observed effects. Thus, to identify MPK3/MPK6 substrates, we performed phosphoproteomics analysis using a novel "Prefractionation-Assisted Phosphoprotein Enrichment" (PAPE) procedure (Lassowskat et al., 2013). The PAPE method mitigates some of the difficulties of detecting phosphoproteins, which are typically of low-abundance, in photosynthesizing green plant tissues. Triplicate samples of pooled plant material were collected for the PAPE fractionation, and as illustrated by ProQ diamond phosphoprotein staining (Figure 6A), the PAPE procedure efficiently enriched for phosphoproteins. Four micrograms of the phosphoprotein-enriched proteins were trypsin-digested and each sample measured twice after a 300-min gradient separation by liquid chromatography. To distinguish between early and late (possibly "indirect") MPK3/6 phospho-targets, we collected leaves at $4,5,7$, and $8 \mathrm{~h}$ after DEX treatment (which overlaps with the initial and sustained MAPK activation profile, c.f. Figure 1).

More than 2000 proteins were detected for each fraction ( $v i z$. 4 h: 2733, 5 h: 2264, 7 h: 2621, 8 h: 2596; see Tables S10-S13). Comparison was carried out with Progenesis LC-MS label-free quantitation based on the peak area of the MS1 spectrum and filtered for significant changes in protein abundance $(>2$-fold, $p<0.05)$ between the $\mathrm{MKK} 5^{\mathrm{DD}}$ - and the MKK5 ${ }^{\mathrm{KR}}$-expressing plants in the Col-0 background (Col-0 DD vs. Col-0 KR). While phosphoprotein enrichment is expected to increase detection of the putative MAPK substrates, several MAPK substrates are destabilized upon phosphorylation (Meng and Zhang, 2013) and may therefore exhibit decreased protein levels after being phosphorylated by MAPKs. Thus, putative phosphoproteins that are either up-regulated or reduced in levels when comparing the
Col-0 DD and Col-0 KR are considered. For the $4 \mathrm{~h}$ treatment, 144 proteins of altered abundance compared to the Col-0 KR line are detected; this progressively increased to more than 729 proteins at $8 \mathrm{~h}$ (Figure 6B, Tables S10-S13). Since the $4 \mathrm{~h}$ time point marks the onset of the MPK3/6 activation (Figure 1), one may expect the 144 proteins with altered abundances at $4 \mathrm{~h}$ (i.e., 73 downand 71 up-regulated) to represent the initial MPK3/6 targets.

For simplicity, we will concentrate here only on the phosphoenriched proteins with increased abundance in the MKK5 ${ }^{\mathrm{DD}}$ plants. Besides the general increase in number of enriched proteins, the protein compositions also changed between the time points-with 44\% (4h), 71\% (5h), 59\% (7h), and 76\% (8h) of the putative phosphoproteins being unique to each time point (Figure 6C). Although not all the putative phosphoproteins detected are direct MAPK substrates and particularly those from the later time points are likely "indirect" phosphoproteins, the above observation suggests a progressive change in the phosphoproteome after simulated MAPK activation. Based on their gene ontology (GO) classifications (Figure 6D), putative phosphoproteins from the $4-5 \mathrm{~h}$ time points are enriched for functions in "transport mechanisms"-in particular vesicle-mediated and nucleocytoplasmic transport. This is in agreement with rapid movement of activated MAPKs into the nucleus (Ligterink et al., 1997; Lee et al., 2004) and observed accumulation of secreted defense metabolites (Figure 2). This is followed (starting at $7 \mathrm{~h}$ ) by proteins with functions known to be regulated by MAPKs, such as embryo development, stress and immune responses. The "later" proteins from the 7-8 h time points appear to be responsible for supplying the biosynthetic precursors and the energetic requirement for production of the numerous defense secondary metabolites. The appearance of components of vacuolar transport and catabolic processes (at 7-8 h) also corroborate the eventual onset of cell death.

Overall, the above analysis revealed a phosphoproteome reprogramming through MPK3/6 activation. These phosphoproteins (consisting of both direct MAPK substrates and other "indirect" phosphoproteins) for the $4-8 \mathrm{~h}$ time points are listed in Figures S10-S13. To facilitate an initial distinction of potential substrates, all phosphoproteins lacking the typical "S/T-P" MAPK-targeted phosphosites are marked in gray in the tables and number of "S/T-P" sites in the putative MAPK targets is indicated. A selection of phosphoproteins with putative functions in plant defense is listed in Table 1, which includes known MAPK substrates. Examples are the Tandem Zinc Finger 7 (TZF7, previously identified as an in vitro MPK3/6 substrate; Feilner et al., 2005), Nitrate Reductase 2 (NR2, an enzyme required for nitric oxide production; Wang et al., 2010), WRKY33 (a transcription factor involved in camalexin production; Mao et al., 2011) or MVQ1 (a VQ-motif-containing protein recently shown to control WRKY-regulated defense gene expression; Pecher et al., 2014). In addition, several WRKYs and gluthathione- $S$-transferases (GSTs) were also found to be putative MAPK substrates.

Interestingly, besides the already mentioned WRKY33 transcription factor, we also find the cytochrome P450 enzymes, CYP71A13 or CYP71B15 (also known as PAD3), as well as members of the non-host penetration resistance pathway, PEN1 to PEN4 (also known as PCS1, Phytochelatin Synthase 1) 


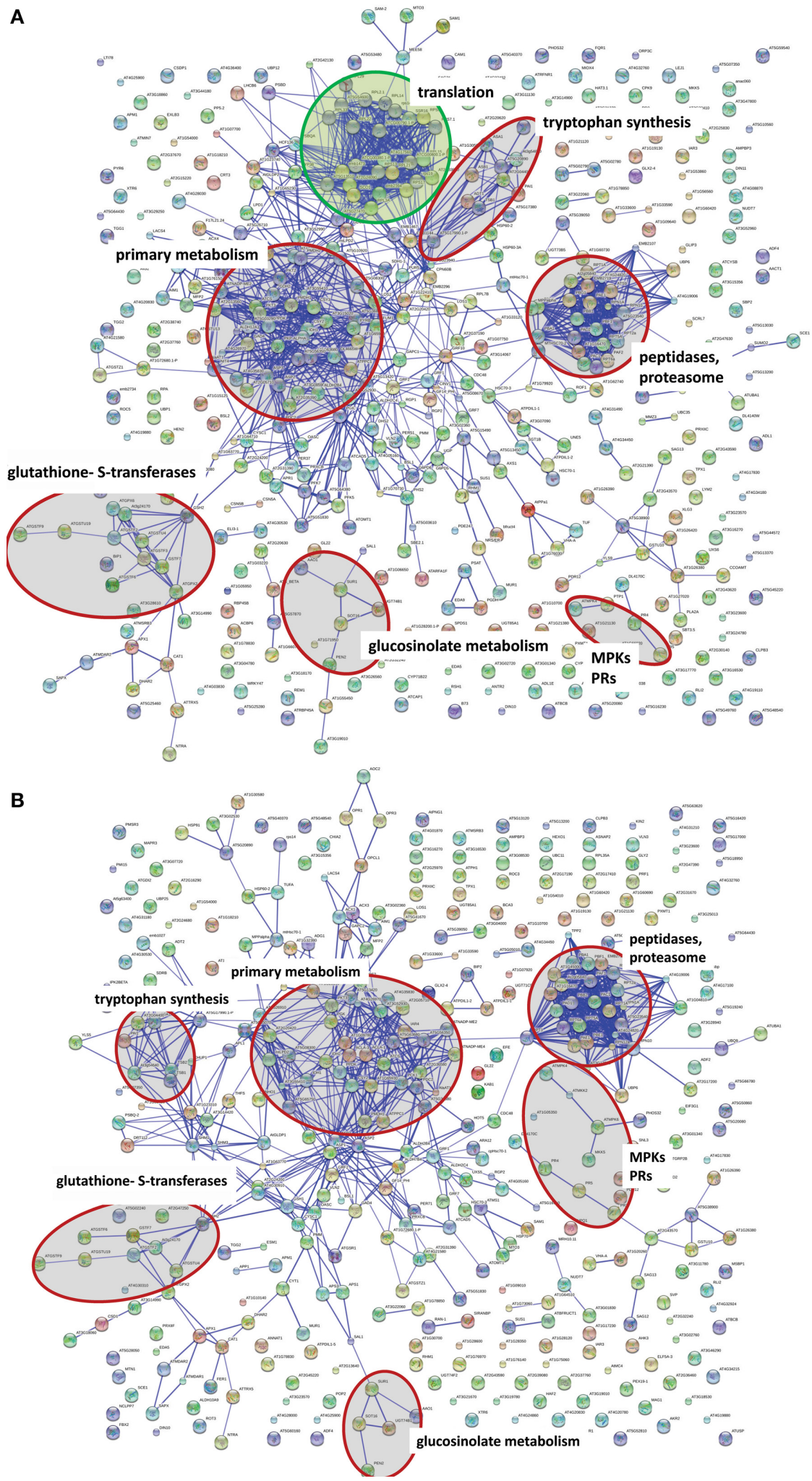

FIGURE 5 | Network comparison of protein extract between wild type DD and $\boldsymbol{m} \boldsymbol{p} \boldsymbol{k}$ 3. DEX treatment causes a wide range of protein changes that can be organized in networks. Network calculation was done with STRING 9.05 (high confidence score 0.7, confidence view). Either the Arabidopsis locus identifier or the protein name abbreviation (where known) are shown. Selected functional clusters are highlighted in red. STRING network of the up-regulated protein in Col-0 DD plants (A) and the mpk3 DD plants (B). The green circle marks a cluster of proteins involved in protein translation, which is completely missing in the mpk3 mutant. 
A
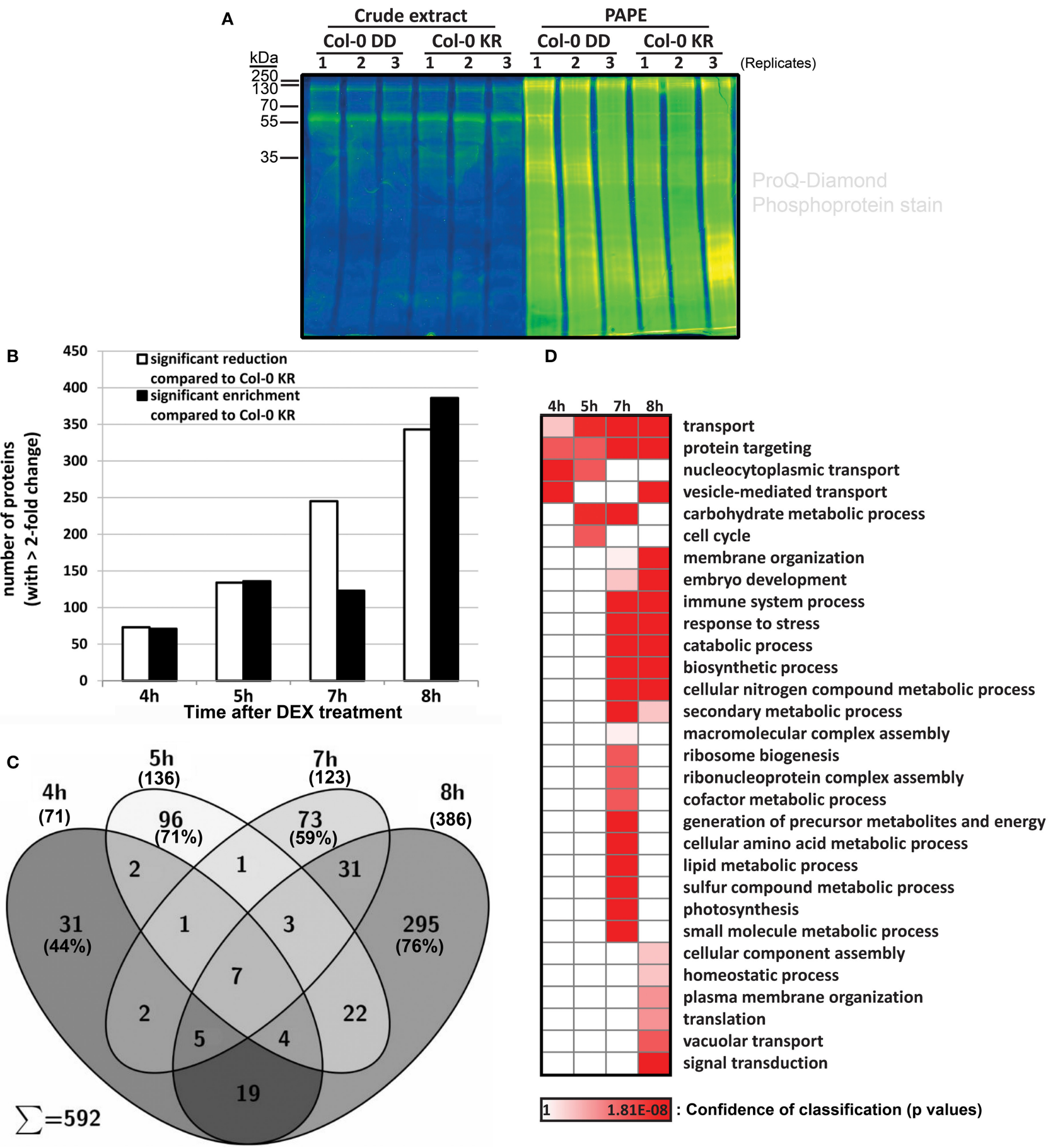

FIGURE 6 | Isolation and identification of phosphoproteins with the prefractionation-assisted phosphoprotein enrichment (PAPE) method. Six week old plants of Col-O DD and control (KR) were treated with DEX, harvested at different time points $(4,5,7,8 \mathrm{~h})$ and subjected to PAPE procedure to isolate phosphoproteins. Analysis of these samples was performed with mass spectrometry and subsequent evaluation with Progenesis LC-MS. (A) Pro-Q Diamond phosphoprotein staining of $\sim 10 \mu \mathrm{g}$ of protein extracts before and after the PAPE procedure. The samples from the $5 \mathrm{~h}$ treatment were used exemplarily to illustrate the efficiency of the phosphoprotein enrichment through PAPE. (B) Number of proteins that

showed a significant enrichment (black bars) or reduction (white bars) during PAPE compared to control plants (ANOVA $p<0.05$ and maximum fold change of $>2.0$ ). (C) Venn diagram showing distribution of proteins with increased abundance (i.e., corresponding to black bars in B) during different time points after DEX treatment. (D) Enrichment of Gene Ontology (GO) biological process classifications in PAPE-protein extract for the putative phosphoproteins with increased abundance over the four time-points after DEX treatment. Annotation and calculation was done with STRING 9.05 ( $p$-value with FDR correction $<0.1$, slim display). The confidence for the enrichment of the GO groups is indicated by the heat map of its $p$-value. 
Table 1 | Selected putative MAPK substrates and phosphoproteins with potential roles in plant defense.

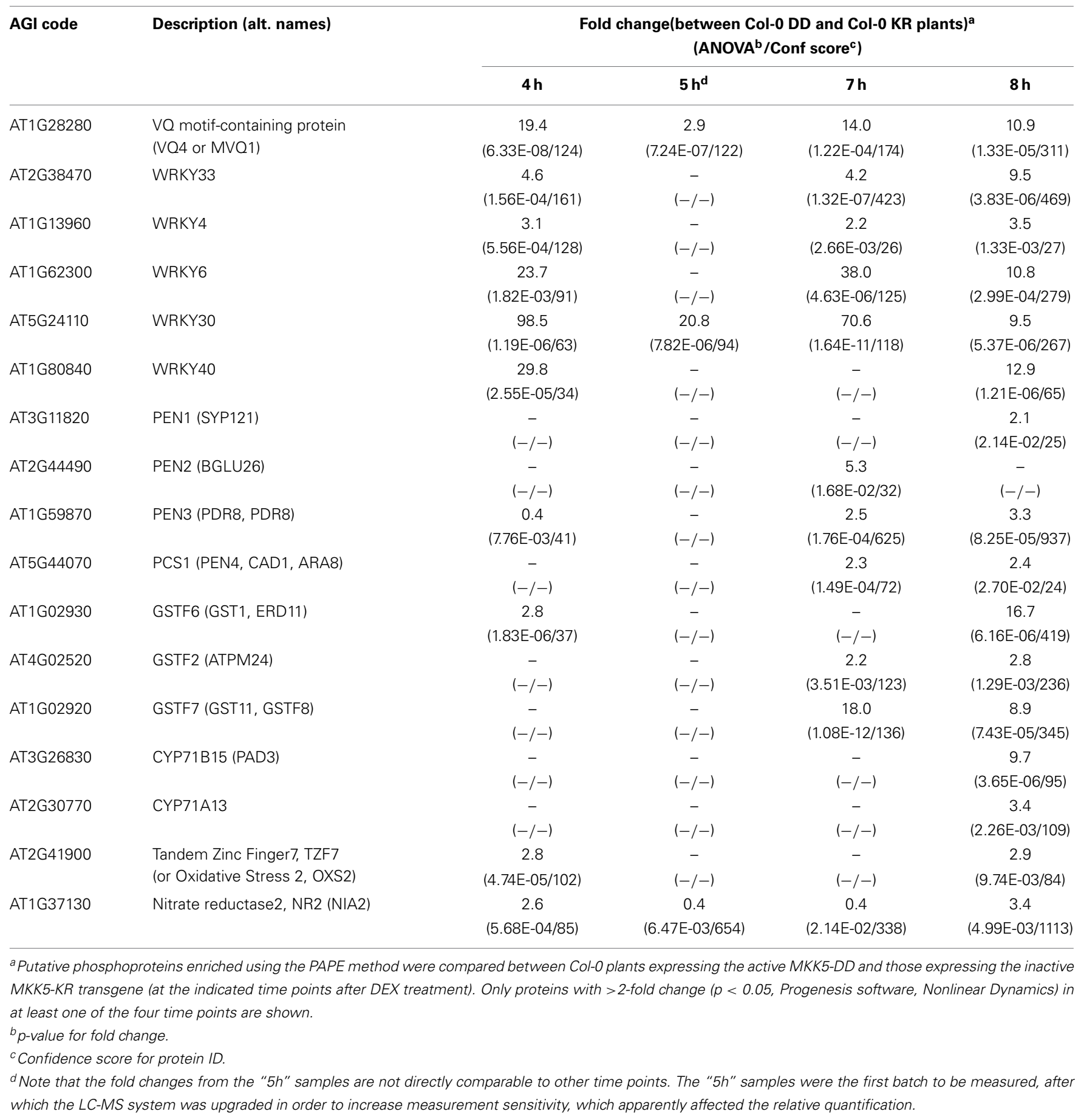

(Gigolashvili and Kopriva, 2014) amongst the enriched putative phosphoproteins. Thus, the increased presence of these enzymes/proteins (presumably in their phosphorylated forms) correlates with the observed accumulation of camalexin and glucosinolate-derivatives (in Figure 2), which supports the notion of increased biosynthesis. Taken together, we could enrich for a number of putative phosphoproteins after an artificial activation of $\mathrm{MPK} 3 / 6$. Since some of these contain typical
MAPK-targeted phosphosites, they represent potential direct MAPK substrates.

\section{VALIDATION OF MAPK SUBSTRATES}

A thorough validation through independent phosphorylation assays would be required to differentiate between the direct MPK3/6 targets and those "indirect" phospho-targets arising from increased protein abundance or through the actions of 
other kinases activated downstream of the MAPKs. As proof-ofprinciple, we selected a few candidates and could confirm that WRKY6, WRKY30 and WRKY33 were indeed used as substrates by MPK6 in an in vitro kinase reaction (Figure 7A).

Another simple but indirect assessment of whether a protein can be potentially phosphorylated is to compare its abundance profile before and after the PAPE step. For instance, in contrast to GSTF2, the enrichment after the PAPE procedure for GSTF7 (4-8 h) does not mirror its overall expression profile (0-24h). Similarly, levels of PEN2 and PEN4/PCS1 appear to be constant or only slightly changed over the $24 \mathrm{~h}$ period but are detected in the phosphoprotein enriched fractions at 7 and $7-8 \mathrm{~h}$, respectively (Figure 7B). This would support the notion that these proteins were indeed phosphorylated (albeit not necessarily by MPK3 or MPK6 directly). A weakness of this method is the requirement of detection in the total proteome analysis but this is often challenging if these proteins are of insufficient abundance in the crude extracts.

Obviously, the most direct evidence would be the identification of the phosphopeptides that match typical MAPK targeted phosphosites. We consolidated all the phosphopeptides identified in all the PAPE samples, which resulted in in 1220 high confidence phosphopeptides (Proteome Discoverer 1.4, false discovery rate adjusted $q<0.05)$ and another 1600 putative phosphopeptides $(q<0.05$ but $>0.01)$ (Table S16) and filtered for phosphopeptides with typical MAPK-targeted SP or TP residues. This revealed $62,17,27$, and 137 peptides with phosphorylated SP or TP residues for the $4-8 \mathrm{~h}$ samples, respectively (Table S17). For illustration, five putative MAPK substrates and their associated phosphopeptides are shown (Figure 7C). Some phosphopeptides were only detected in the Col-0 DD plants but not the Col$0 \mathrm{KR}$ plants, suggesting these are phosphorylated after MPK3/6 activation (e.g., MVQ1, TZF7, and WRKY6). A few other phosphopeptides pre-existed in selected time points of the Col-0 KR plants, which meant they were already phosphorylated prior to the MPK3/6 activation (e.g., one phosphopeptide for NR2 or some phosphopeptides in the tetratricopeptide repeat (TPR)-like protein encoded by $A t 4 g 28080$, Figure 7C). In addition, we also checked for these phosphopeptides in plants with the MKK5 $5^{\mathrm{DD}}$ transgene in $m p k 3$ or $m p k 6$ background. The similar or differential detection of these phosphopeptides in these mutant backgrounds indicate either redundancies between the two MAPKs or preferential dependence on one of the two MAPKs in mediating the phosphorylation, respectively (Figure 7C). For instance, detection of MVQ1 phosphopeptides is absent in the mpk6 background (Figure 7C), which is in agreement with recent data from our laboratory that MPK6 is the major in vivo kinase for MVQ1 after MAMP elicitation (Pecher et al., 2014).

To validate the detected phosphosites, we also performed a targeted proteomics analysis where an "inclusion list" of the desired phosphopeptides (Table S20) was used to enable relative quantification of the phosphosites within each protein. For 17 of these putative substrates, the phosphopeptides with higher peptide spectral match (PSM) scores mark the relatively most abundant in vivo phosphosites (Table 2). For instance, although previously shown to be multiply phosphorylated at nearly all its potential phosphosites (Pecher et al., 2014), the S235 and S215 residues in the C-terminus of the MVQ1 protein appear to be the most prominent in vivo phosphosites (Table 2). Similarly, the predominant in vivo phosphosites could be identified for three known in vitro MPK3/6 substrates (TZF7, WRKY6, and NR2), as well as 13 other novel MPK3/6 targets using the targeted proteomics approach (Table 2 ). In summary, our phosphoproteomics approach enabled several known and numerous novel candidate MAPK substrates that are potentially involved in defense regulation to be identified.

\section{DISCUSSION}

\section{SUSTAINED MAPK ACTIVATION IS SUFFICIENT TO DRIVE ANTIMICROBIAL METABOLITE ACCUMULATION}

In this work, we "hijacked" the MAPK pathway by introducing a constitutively-active MKK5 to mimic pathogen- or stressmediated MPK3/6 activation. Since MPK3 and MPK6 are also involved in various developmental processes such as leaf abscission and development of stomata, anthers or embryos (Wang et al., 2007; Cho et al., 2008; Hord et al., 2008; Wang et al., 2008), such an artificial MAPK activation may lack proper regulation and one may question its physiological relevance. However, our system is focused on a specific developmental stage and tissue (i.e., 6-week-old leaves) within a defined time period. Hence, the effects of MPK3/6 activation for the abovementioned developmental processes are of little bearing in this system. In contrast to the typical transient MAPK activation after MAMP treatment (typically lasting for less than $60 \mathrm{~min}$ ), the use of the dexamethasone induction here causes a prolonged MPK3/6 activation. Our metabolite profiling showed that such sustained MPK3/6 activation elicited a massive reprogramming of the defense metabolome, where we found high accumulation of camalexin, indole glucosinolate derivatives (Figure 2, Figures S2A-C). Additionally, levels for many defense-related phytohormones such as JA, SA (Figures S2G,H), and ET (Han et al., 2010) were also elevated (albeit tissue damage may account for accumulation of some of the "late" metabolites). By contrast, flg22 elicitation, which causes only transient MAPK activation, led to accumulation of comparatively low levels of camalexin (Millet et al., 2010; Schenke et al., 2011). Thus, the system used is comparable to the extended MAPK activation effected during effector-triggered immunity as compared to PTI. Recent work also suggested that sustained MAPK activation is "a critical determinant for modulation of robustness of the immune signaling network" (Tsuda et al., 2013). A conclusion of the metabolome analysis presented here is that sustained MAPK activation is sufficient, and apparently the key determinant, for reprogramming cellular metabolic pathways into antimicrobial and defense metabolite production.

\section{ROLE OF ROS FROM RBOHD FOR DEFENSE METABOLITE ACCUMULATION AND CELL DEATH}

Manipulating in vivo phytoalexin production can be a way to engineer pathogen-resistant plants. However, the sustained MAPK activation also caused cell death that would be counterproductive for plant growth and crop yield (Figure 1). Since ultimate cell death was partially reduced in the ein3/eill and 
A

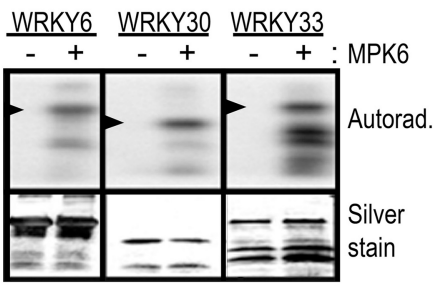

B
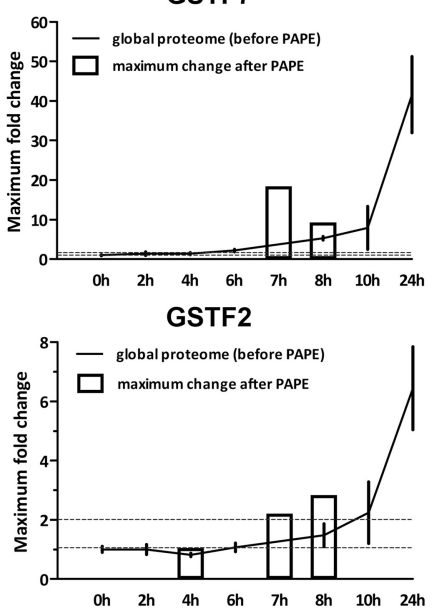

PEN2

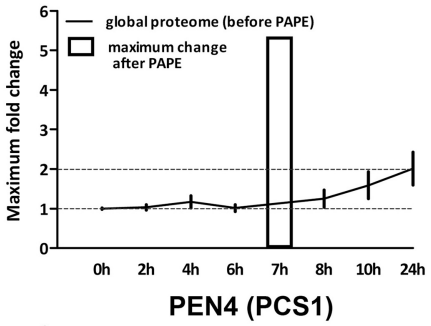

$\left.{ }^{4}\right]$ - global proteome (before PAPE)

$\square$ maximum change after PAPE

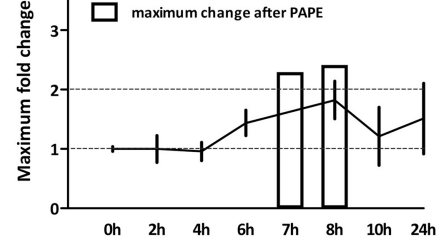

C

AT1G37130.1 - nitrate reductase 2 (NR2) SDsPKAHQNQTTNQTVFLKPAK

SYKPPVPGRSDSPK

IGELITTGYSSDSSSPNNSVHGSSAVFSLLAPIGEATPVR

AT1G28280.1 - VQ motif-containing protein, MVQ1 (or VQ4) LLPLFPVTSPR

GFYLHPSPATTPMDPEPR

INPLNPVFNPVNSAFSPR

SGSSNQSPNELAAEEK

KPEILSPSILDFPSLVLSPVTPLIPDPFDR

AT2G41900.1 - TZF7

NVVEPISPMSAR

FTDSALASAVFsPTHK

SVDHSLFSGGGRMsPR

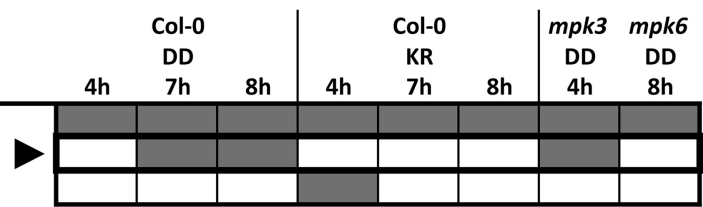

AT4G28080.1 - Tetratricopeptide repeat (TPR)-like superfamily protein

SALASSACTEQINKPTPMLSPVSVK

MFVKsPLNKK

VVVGGSELTSSPKEIKNVEVEK

TVDGETENLPNGDSSPKSSVAADGEK

VVVGGSELTSSPK

TVDGETENLPNGDSsPK

MFVKsPLNK

GKPGQSPGPVSEENQKDDEILSPAHLTGESSSDKENK

SAQMAEDSDTCLLNKSPTANDSNGSESVIGVK

KESGSTDGDSPTEKDAGDSNSGLSPKPK

STNFTSPR

QNNSSVVGERPVNDKSALASSACTEQINKPTPMLSPVSVK

ESGSTDGDSPTEKDAGDSNSGLSPKPK

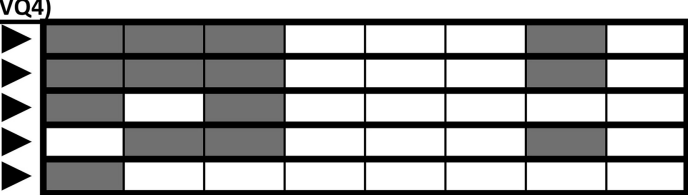

AT1G62300.1 - WRKY6

LGREESPETESNKIQK

FIGURE 7 | Validation of phosphorylation of the identified putative substrates. (A) In vitro kinase assay using recombinant MPK6 and recombinant His-tagged WRKY proteins as substrates in the presence of $\gamma$-32P-ATP. Phosphorylated substrates are visualized by phosphor-imaging (top panel) and equal loading determined by silver staining of the gels (bottom panel). Arrowheads mark the position for the expected size of the full-length proteins (smaller protein bands are presumably from partial or degradation products). (B) Comparison of protein levels before and after the PAPE procedure for selected putative substrates/phospho-proteins. The relative fold phospho-protein enrichment after PAPE (between the
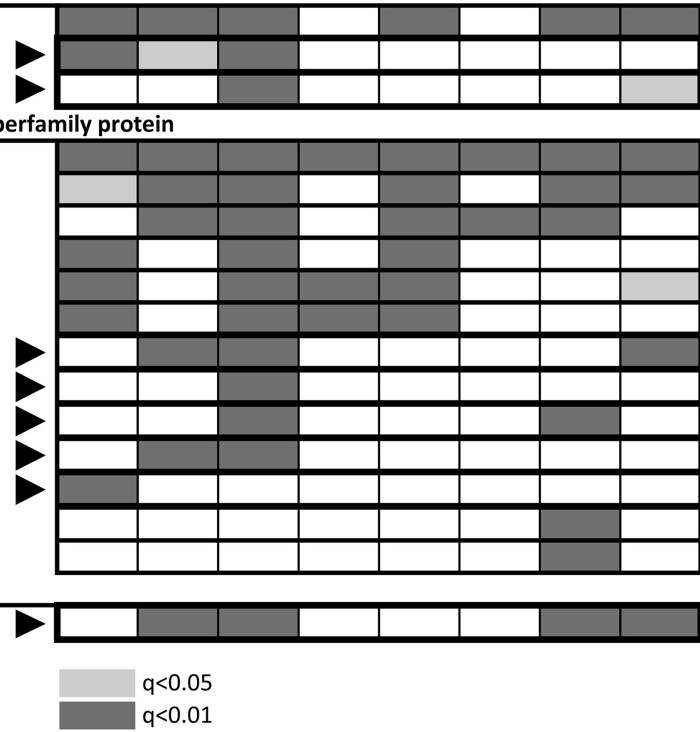

Col-0 DD and the Col-0 KR plants) for the 4, 7, and $8 \mathrm{~h}$ time points after DEX treatment (white bars) were superimposed over the protein levels detected over the $24 \mathrm{~h}$ global proteome analysis. (C) Phosphorylation of SP/TP motifs within selected putative MAPK substrates. Proteome Discoverer 1.4 was used to consolidate the data from different experiments. The shadings denote the detection of the indicated phosphopeptides (gray: $q<0.01$ or light gray: $q<0.05$ ). Black arrowheads mark the phosphopeptides that are only detected in the Col-0 DD but not the KR lines. The phosphorylated residues are indicated as small letters within the peptide sequence. 


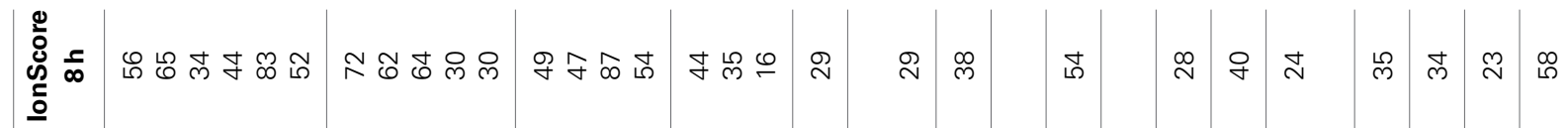

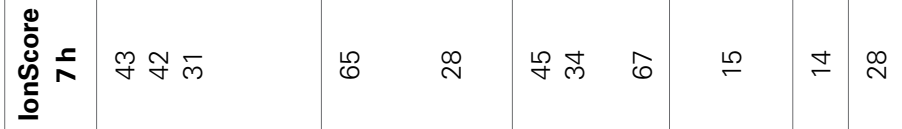
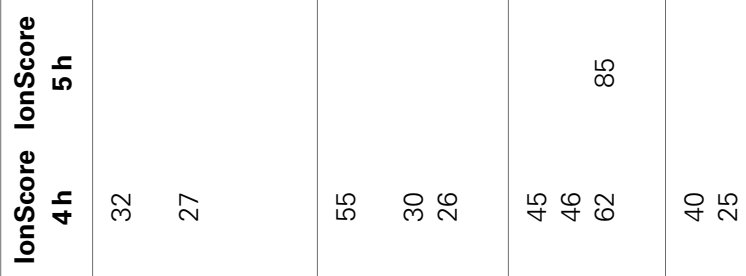

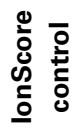

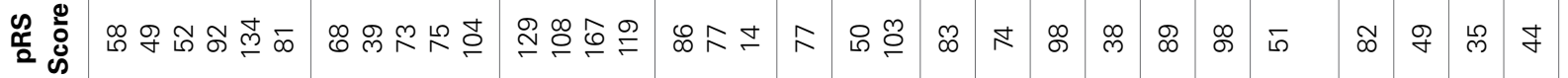

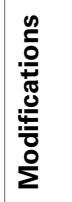

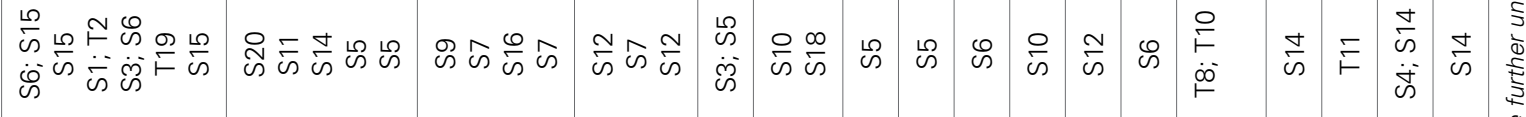

舟

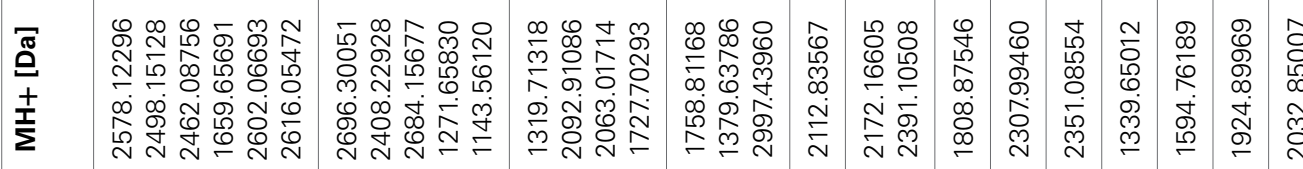

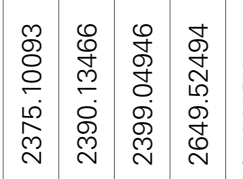

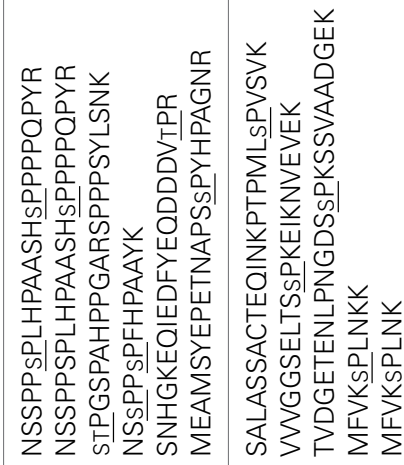
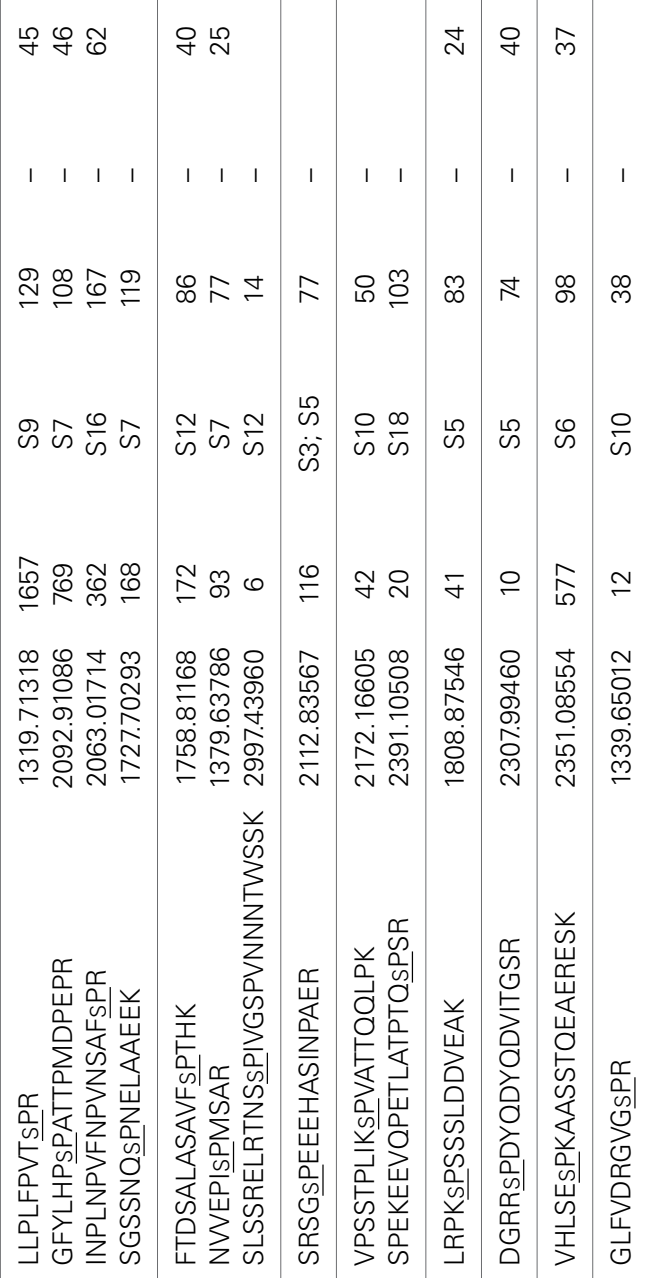
เே

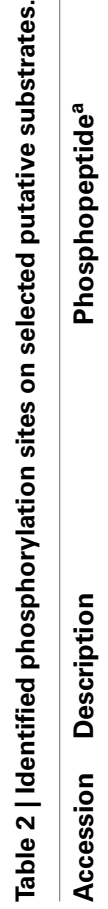

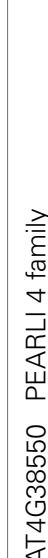
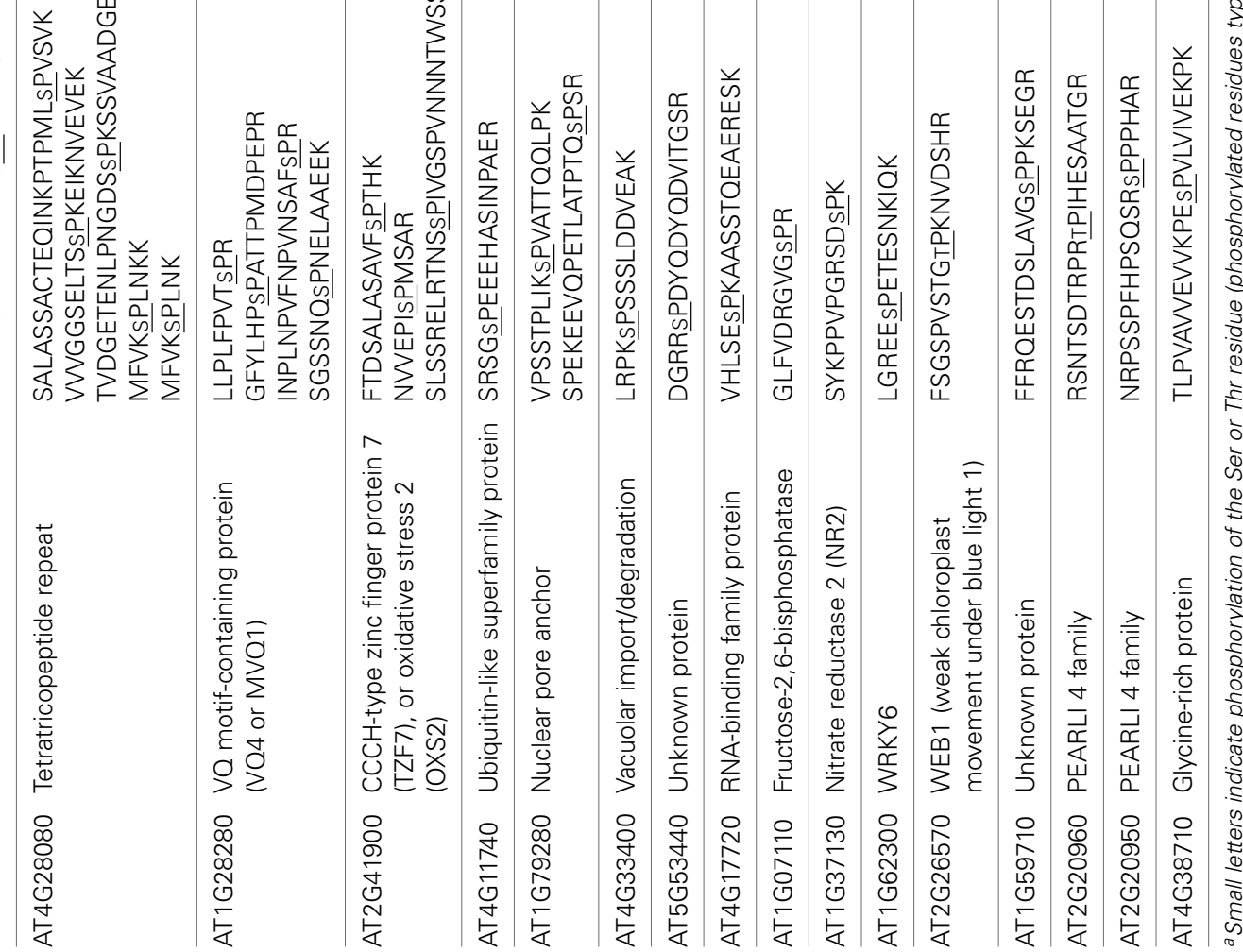

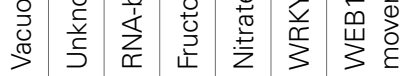

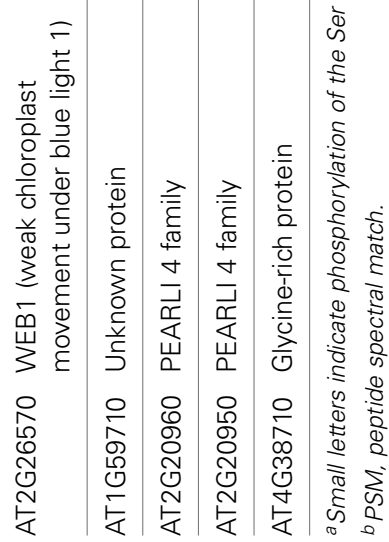


rbohD mutants (Figure 1), we compared the observed phenotypes in the different tested genotypes. Overall, MPK6 appears to be more important than MPK3 in mediating accumulation of the tested defense metabolites (Figures 3A-C). While acetylagmatine required either ethylene or ROS generated by RBOHD (Figure 3C), accumulation of the Trp-derived antimicrobial substances are independent of ethylene signaling (Figures 3A,B). $\mathrm{RBOHD}$ is, however, required for camalexin accumulation. The related $\mathrm{RBOHF}$ was previously thought to play a stronger role than RBOHD for camalexin accumulation induced by introducing a mutated catalase allele (Chaouch et al., 2012). Although we did not investigate RBOHF directly, RBOHF activity is still present in the rbohD mutant used here and therefore suggests RBOHD activity (instead of RBOHF) being essential for camalexin and acetylagmatine accumulation. Our findings are therefore in agreement that RBOHD is the major contributor for MAMP-induced ROS (Mersmann et al., 2010; Ranf et al., 2011). A conflict with previous studies is that the ROS generated in such chemically-induced MAPK plants has been reported to be of chloroplastic origin (Liu et al., 2007). Interestingly, while cell death induction is seen in all tested genotypes, new rosette leaves emerged from the apical meristem of the rbohD plants (Figure 1), indicating that ROS is needed for complete cell death. Thus, besides chloroplastic ROS, RBOHD-generated superoxide is also important. Cell-to-cell propagation and amplification of ROS mediated by RBOHD (Miller et al., 2009; Dubiella et al., 2013) could account for the penetrance of cell death induction into the deeper meristem tissues. Since this ROS propagation circuit is mediated by RBOHD phosphorylation through the calciumdependent protein kinase, CPK5 (Dubiella et al., 2013), it raises the question if the elevated MAPK activities in our system may crosstalk with the CPK5 pathway. Taken together, accumulation of defense compounds and cell death are intertwined and further studies are needed to separate these two processes in order to exploit this knowledge for increasing plant resistance without adverse penalties for crop yield.

\section{DIFFERENCES BETWEEN MPK3 AND MPK6}

The aim of this study is to identify downstream phospho-targets (including direct substrates) of MPK3 or MPK6. To identify specific MPK3 or MPK6 substrates, we also compared the PAPEfractionated phosphoproteins from $m p k 3$ and $m p k 6$ backgrounds to the "core" set of experiments based on the Col-0 genotype. A single time point was chosen for this analysis. Due to slightly variable expression profiles of the transgene in the different genotypes, we decided on $4 \mathrm{~h}(m p k 3)$ or $8 \mathrm{~h}$ (mpk6) after DEX treatment for comparison to the Col-0 background. This timing was based on a pilot study of the induction profile of the $\mathrm{MKK} 5^{\mathrm{DD}}$-expression in the different genotypes. Comparison of the resulting protein lists showed that most of the enriched phosphoproteins in Col-0 were reduced in levels or missing in the mpk6 background (Figure S3, Table S15), suggesting it is the major kinase for the differentially detected phosphoproteins. Specific examples include WRKY6, WRKY30, WRKY33, and WRKY40 (Figure S3B). By contrast, abundance of putatively phosphorylated WRKY3, WRKY6, and WRKY33 (at $4 \mathrm{~h}$ post DEX) in the mpk3 mutant is mostly like in Col-0 plants
(Figure S3A). Despite this apparent reliance on MPK6 activity, the $m p k 3$ mutant also had many unique putative phosphoproteins (i.e., more abundantly detected in the $m p k 3$ mutant compared to Col-0; see lower half of Table S15A). Taken together with the global proteome data that a cluster of proteins involved in translation is missing in the mpk3 mutant (Figure 5), as well as the previously reported haploinsufficiency of MPK3 in ovule development (Wang et al., 2008), there is differential preference for the various substrates by the two MAPKs, and they are not totally redundant as often thought. A caveat of the above phosphoproteomics comparative analysis is that only a single timepoint was chosen; future studies should preferably include a time course series (as done for the "core" experiment) to allow for variance in induction profiles.

\section{COMPARISON TO OTHER PROTEOMICS STUDIES OF MAPK SUBSTRATE IDENTIFICATION}

In a similar analysis to uncover candidate MPK3/6 substrates, metal-oxide affinity chromatography was used to enrich for phosphopeptides in Arabidopsis expressing the tobacco MKK5 ${ }^{\mathrm{DD}}$ variant (Hoehenwarter et al., 2012). Besides the origin of the MKK5 construct, another difference to this work is the tissue type and developmental stage employed: they used 12day-old seedlings liquid-grown in continuous light while our plants are 6-week-old adult soil-grown plants under standard short day light regime. Potential substrates required for rapid response to pathogen attack are presumably pre-existing in leaves as hypo-phosphorylated forms. We may assume that using adult leaves bears more potential in finding substrates relevant for the pathogen response under natural growth conditions. Furthermore, Hoehenwarter et al. (2012) compared seedlings with or without DEX treatment while we used the expression of a kinase-inactive $\mathrm{MKK} 5^{\mathrm{KR}}$ variant to exclude any impact arising from the expression of the MKK5 protein alone. Their list of candidate MPK3/6 substrates contains 140 proteins. Our consolidated list from the four time points $(4-8 \mathrm{~h})$ yielded 539 putative phosphoproteins (Table S14). Thirty-seven of these proteins are common between our list and that of Hoehenwarter et al. (2012) (Figure S4, Table S18), which includes known MPK3/6 substrates such as TZF7, NR3, MVQ1 (VQ4), and WRKY40 (Feilner et al., 2005; Popescu et al., 2009; Wang et al., 2010; Pecher et al., 2014). The MAPK phosphatase, MKP1, a reported substrate of MAPKs (Gonzalez Besteiro and Ulm, 2013) and the universal stress proteins PHOS32/34 (Merkouropoulos et al., 2008) are found in the 103 candidates unique to the "Hoehenwarter" list. Similarly, in the candidates found exclusively in our list are also known MAPK substrates such as the chromatin remodeling ATPase, BRAHMA (Feilner et al., 2005), decapping complex protein1, DCP1 (Xu and Chua, 2012), WRKY6 (Popescu et al., 2009), and WRKY33 (Mao et al., 2011) (Figure S4). Thus, both studies enabled bona fide MAPK substrates to be identified; but the differences in the studies also highlight that each study has its strengths or weaknesses.

One of the strength of the Hoehenwarter et al. (2012) work is the direct detection of the SP or TP residues in the phosphopeptides. We were also able to identify many phosphopeptides with phosphorylated SP or TP residues (Table S17) but for other 
candidates, direct detection of such sites were not found. The latter is likely due to the fact that our work is based on phosphoprotein enrichment and it is difficult to detect low abundance phosphopeptides in the complex peptide mixtures reintroduced after tryptic digest of the proteins. One consideration for the future is to add another phosphopeptide enrichment step after the PAPE procedure to enhance phosphopeptide detection sensitivity. Nevertheless, the advantage of our analysis is that our list yielded a larger number of candidates for future analysis. Of course, the larger number of candidates we obtained may also be accounted by the use of a range of time points for the DEX treatment, which may cover early and late substrates. Since some MAPK substrates are destabilized upon phosphorylation (e.g., MVQ1, Pecher et al., 2014), their detection in the current experimental set-up is a balance between the rate of protein decay and efficiency of the phosphoprotein enrichment. In fact, we observed some fluctuations within the $4-8 \mathrm{~h}$ time course in abundance of some of the candidates (Table S14) or the detection of specific phosphopeptides (an example is At4g28080, see Figure 7B). This may reflect a change in the protein destabilization rate and therefore usage of several time points enhances the detection window and improves the chance of discovery. In this respect, one should also consider the candidate phosphoproteins that are down-regulated in the $\mathrm{MKK} 5^{\mathrm{DD}}$ line compared to the MKK $5^{\mathrm{KR}}$ line (Figure 6B, Tables S10-S13).

\section{IDENTIFICATION OF KNOWN AND POTENTIAL NOVEL MAPK SUBSTRATES}

Identification of known MAPK substrates in the consolidated list of the enriched phosphoproteins (4-8 h after DEX treatment), such as MVQ1, NR2, TZF7, VIP1, and several WRKYs (Feilner et al., 2005; Djamei et al., 2007; Wang et al., 2010; Mao et al., 2011; Maldonado-Bonilla et al., 2014; Pecher et al., 2014), confirmed that the artificial MAPK activation system coupled to an efficient phosphoprotein enrichment method (Lassowskat et al., 2013) can identify direct MPK3/6 substrates that regulate plant immunity responses. Among the potential novel substrates and downstream "indirect" phosphoproteins, we want to particularly highlight three functional groups that deserve future attention:

(I) The first is a group of processing-body (P-body) components such as the mRNA decapping complex proteins, DCP1 and VARICOSE (VCS) and the exoribonuclease, XRN4 (Xu and Chua, 2010, 2012). We also found TZF7 as an enriched putative phosphoprotein. Recently, we showed that the related TZF9 is an MPK3/6 substrate and colocalizes to P-bodies (Maldonado-Bonilla et al., 2014). Remarkably, the putative RNA ligase/cyclic nucleotide phosphodiesterase family protein, encoded by At3g28140, which was found as an interacting partner of both TZF7 and TZF9 in a large-scale interactome study (Arabidopsis Interactome Mapping, 2011), was also phospho-enriched in the $7 \mathrm{~h}$ sample (Table S14). Taken together with the report on MPK6 phosphorylation of DCP1 (Xu and Chua, 2012), there is rising evidence that MAPKs affect posttranscriptional gene regulation through elements of mRNA processing in P-bodies.
(II) The second group includes proteins such as exocyst components (Exo70B2, Exo70E1, Sec3A, Sec5A), COPII components of ER-associated vesicles (Sec23/Sec24), the Sec7 guanine exchange factor, the SecY translocon, or several clathrin-related proteins. These proteins are involved in vesicle/membrane transport or secretion, and therefore may mediate the observed secretion of defense metabolites and presumably shuttling of key defense proteins in various membrane compartments.

(III) A third group of proteins are those that may be responsible for the indole glucosinolate and camalexin accumulation. PAD3 is a camalexin biosynthetic enzyme, whose expression is regulated by WRKY33 (Mao et al., 2011). The detection of the PEN1-PEN4 proteins around 7-8 h after DEX treatment (Table 1) is just slightly trailing behind the increase of various indole glucosinolate derivatives (typically $<6$ h, Figure 2). Nevertheless, the rapid increase in the indole glucosinolate metabolites is indicative for increased activities of these PEN proteins. Although PEN1, PEN3, and PEN4/PCS1 are known phosphoproteins (Nühse et al., 2003; Stein et al., 2006; Benschop et al., 2007; Wang et al., 2009), many of the reported phosphosites are not the typical MAPK targeted sites; thus it is unclear if these are direct MAPK targets or detected due to increased protein abundance. In the case of PEN2, it was highly phospho-enriched (at $7 \mathrm{~h}$ after DEX treatment) although there was no apparent increase in expression in the global proteome analysis (see comparison in Figure 7B). Hence, PEN2 is a candidate phospho-target after MAPK activation. This raises the possibility that activities of PEN proteins are regulated by phosphorylation through MAPKs or other kinases, which must be tested in the future.

\section{CONCLUDING REMARKS}

In conclusion, this work provided a catalog of potential phosphoproteins downstream of MPK3/6 activation, including direct MPK3/6 substrate candidates. The importance of sustained MPK3/6 activation in plant chemical defense regulation is uncovered through a parallel metabolomics analysis that revealed a massive reprogramming of defense metabolism-in particular accumulation of camalexin, indole glucosinolate, agmatine and a variety of their derivatives. Several of the detected phosphoproteins/MAPK substrate candidates are involved in the corresponding regulatory (or biosynthesis) pathways and may account for the production of these defense compounds. Thus, besides re-programming of chemical defense, and as already discussed above, many of the other putative MAPK substrates or "indirect" phosphoproteins are components of RNA metabolism or secretory pathways. Notably, there is so far only limited information on MAPK-mediated control of the latter two processes. This work therefore identifies the putative phosphoproteins as a basis for elucidating the MAPK-mediated regulon of plant defense against pathogens or abiotic stresses. Future work will focus on uncovering the impact of phosphorylation on the activities or function of these phospho-proteins or MAPK substrates.

Dataset: The proteomics data have been deposited to the ProteomeXchange Consortium (Vizcaíno et al., 2014) 
(http://proteomecentral.proteomexchange.org) via the PRIDE partner repository with the dataset identifier PXD001252 and DOI 10.6019/PXD001252.

\section{ACKNOWLEDGMENTS}

Pascal Pecher performed the in vitro kinase assay for the WRKY proteins. We thank Dr Kai Naumann for advice on experimental design and management of the proteomics mass spectrometer facility, Nicole Bauer, Petra Majovsky, and Sylvia Krüger for technical assistance, and members from our laboratory for insightful discussions. This work is financed by the German Research Foundation through SFB648 (Molecular mechanisms of information processing in plants) and the ERA-PG project "PathoNET" (SCHE 235/15-1) to Dierk Scheel and Justin Lee. The BMBF project ProNET-T3 (03ISO2211B) supported Lennart Eschen-Lippold.

\section{SUPPLEMENTARY MATERIAL}

The Supplementary Material for this article can be found online at: http://www.frontiersin.org/journal/10.3389/fpls.2014. 00554/abstract

\section{REFERENCES}

An, F., Zhao, Q., Ji, Y., Li, W., Jiang, Z., Yu, X., et al. (2010). Ethylene-induced stabilization of ETHYLENE INSENSITIVE3 and EIN3-LIKE1 is mediated by proteasomal degradation of EIN3 binding F-box 1 and 2 that requires EIN2 in Arabidopsis. Plant Cell 22, 2384-2401. doi: 10.1105/tpc.110.076588

Andreasson, E., and Ellis, B. (2010). Convergence and specificity in the Arabidopsis MAPK nexus. Trends Plant Sci. 15, 106-113. doi: 10.1016/j.tplants.2009.12.001

Andreasson, E., Jenkins, T., Brodersen, P., Thorgrimsen, S., Petersen, N. H., Zhu, S., et al. (2005). The MAP kinase substrate MKS1 is a regulator of plant defense responses. Embo J. 24, 2579-2589. doi: 10.1038/sj.emboj.7600737

Aoyama, T., and Chua, N. H. (1997). A glucocorticoid-mediated transcriptional induction system in transgenic plants. Plant J. 11, 605-612. doi: 10.1046/j.1365313X.1997.11030605.x

Arabidopsis Interactome Mapping, C. (2011). Evidence for network evolution in an Arabidopsis interactome map. Science 333, 601-607. doi: 10.1126/science. 1203877

Barth, C., and Jander, G. (2006). Arabidopsis myrosinases TGG1 and TGG2 have redundant function in glucosinolate breakdown and insect defense. Plant J. 46, 549-562. doi: 10.1111/j.1365-313X.2006.02716.x

Bednarek, P., Pislewska-Bednarek, M., Svatos, A., Schneider, B., Doubsky, J., Mansurova, M., et al. (2009). A glucosinolate metabolism pathway in living plant cells mediates broad-spectrum antifungal defense. Science 323, 101-106. doi: $10.1126 /$ science. 1163732

Benschop, J. J., Mohammed, S., O'flaherty, M., Heck, A. J., Slijper, M., and Menke, F. L. (2007). Quantitative phosphoproteomics of early elicitor signaling in Arabidopsis. Mol. Cell. Proteomics 6, 1198-1214. doi: 10.1074/mcp.M600429MCP200

Bethke, G., Pecher, P., Eschen-Lippold, L., Tsuda, K., Katagiri, F., Glazebrook, J., et al. (2012). Activation of the Arabidopsis thaliana mitogen-activated protein kinase MPK11 by the flagellin-derived elicitor peptide, flg22. Mol. Plant Microbe Interact. 25, 471-480. doi: 10.1094/MPMI-11-11-0281

Bethke, G., Unthan, T., Uhrig, J. F., Pöschl, Y., Gust, A. A., Scheel, D., et al. (2009). Flg22 regulates the release of an ethylene response factor substrate from MAP kinase 6 in Arabidopsis thaliana via ethylene signaling. Proc. Natl. Acad. Sci. U.S.A. 106, 8067-8072. doi: 10.1073/pnas.0810206106

Blume, B., Nürnberger, T., Nass, N., and Scheel, D. (2000). Receptor-mediated increase in cytoplasmic free calcium required for activation of pathogen defense in parsley. Plant Cell 12, 1425-1440. doi: 10.1105/tpc.12.8.1425

Boller, T., and Felix, G. (2009). A renaissance of elicitors: perception of microbe-associated molecular patterns and danger signals by pattern-recognition receptors. Annu. Rev. Plant Biol. 60, 379-406. doi: 10.1146/annurev.arplant.57.032905.105346
Chaouch, S., Queval, G., and Noctor, G. (2012). AtRbohF is a crucial modulator of defence-associated metabolism and a key actor in the interplay between intracellular oxidative stress and pathogenesis responses in Arabidopsis. Plant J. 69, 613-627. doi: 10.1111/j.1365-313X.2011.04816.x

Cho, S. K., Larue, C. T., Chevalier, D., Wang, H., Jinn, T. L., Zhang, S., et al. (2008). Regulation of floral organ abscission in Arabidopsis thaliana. Proc. Natl. Acad. Sci. U.S.A. 105, 15629-15634. doi: 10.1073/pnas.0805539105

Clay, N. K., Adio, A. M., Denoux, C., Jander, G., and Ausubel, F. M. (2009). Glucosinolate metabolites required for an Arabidopsis innate immune response. Science 323, 95-101. doi: 10.1126/science.1164627

Djamei, A., Pitzschke, A., Nakagami, H., Rajh, I., and Hirt, H. (2007). Trojan horse strategy in Agrobacterium transformation: abusing MAPK defense signaling. Science 318, 453-456. doi: 10.1126/science.1148110

Dubiella, U., Seybold, H., Durian, G., Komander, E., Lassig, R., Witte, C. P., et al. (2013). Calcium-dependent protein kinase/NADPH oxidase activation circuit is required for rapid defense signal propagation. Proc. Natl. Acad. Sci. U.S.A. 110, 8744-8749. doi: 10.1073/pnas.1221294110

Eschen-Lippold, L., Bethke, G., Palm-Forster, M. A., Pecher, P., Bauer, N., Glazebrook, J., et al. (2012). MPK11-a fourth elicitor-responsive mitogenactivated protein kinase in Arabidopsis thaliana. Plant Signal. Behav. 7, 1203-1205. doi: 10.4161/psb.21323

Feilner, T., Hultschig, C., Lee, J., Meyer, S., Immink, R. G. H., Koenig, A., et al. (2005). High throughput identification of potential Arabidopsis mitogenactivated protein kinases substrates. Mol. Cell. Proteomics 4, 1558-1568. doi: 10.1074/mcp.M500007-MCP200

Franceschini, A., Szklarczyk, D., Frankild, S., Kuhn, M., Simonovic, M., Roth, A., et al. (2013). STRING v9.1: protein-protein interaction networks, with increased coverage and integration. Nucleic Acids Res. 41, D808-D815. doi: 10.1093/nar/gks1094

Gigolashvili, T., and Kopriva, S. (2014). Transporters in plant sulfur metabolism. Front. Plant Sci. 5:442. doi: 10.3389/fpls.2014.00442

Gonzalez Besteiro, M. A., and Ulm, R. (2013). Phosphorylation and stabilization of Arabidopsis MAP kinase phosphatase 1 in response to UV-B stress. J. Biol. Chem. 288, 480-486. doi: 10.1074/jbc.M112.434654

Han, L., Li, G. J., Yang, K. Y., Mao, G., Wang, R., Liu, Y., et al. (2010). Mitogen-activated protein kinase 3 and 6 regulate Botrytis cinerea-induced ethylene production in Arabidopsis. Plant J. 64, 114-127. doi: 10.1111/j.1365313X.2010.04318.x

Hoehenwarter, W., Thomas, M., Nukarinen, E., Egelhofer, V., Röhrig, H., Weckwerth, W., et al. (2012). Identification of novel in vivo MAP kinase substrates in Arabidopsis thaliana through use of tandem metal oxide affinity chromatography. Mol. Cell. Proteomics 12, 369-380. doi: 10.1074/mcp.M112. 020560

Hord, C. L., Sun, Y. J., Pillitteri, L. J., Torii, K. U., Wang, H., Zhang, S., et al. (2008). Regulation of Arabidopsis early anther development by the mitogen-activated protein kinases, MPK3 and MPK6, and the ERECTA and related receptor-like kinases. Mol. Plant 1, 645-658. doi: 10.1093/mp/ssn029

Jabs, T., Tschöpe, M., Colling, C., Hahlbrock, K., and Scheel, D. (1997). Elicitorstimulated ion fluxes and $\mathrm{O} 2$ - from the oxidative burst are essential components in triggering defense gene activation and phytoalexin synthesis in parsley. Proc. Natl. Acad. Sci. U.S.A. 94, 4800-4805. doi: 10.1073/pnas.94.9.4800

Joo, S., Liu, Y., Lueth, A., and Zhang, S. Q. (2008). MAPK phosphorylationinduced stabilization of ACS6 protein is mediated by the non-catalytic Cterminal domain, which also contains the cis-determinant for rapid degradation by the $26 \mathrm{~S}$ proteasome pathway. Plant J. 54, 129-140. doi: 10.1111/j.1365313X.2008.03404.x

Knogge, W., Lee, J., Rosahl, S., and Scheel, D. (2009). "Signal perception and transduction in plants," in Plant Relationships, 2nd Edn., ed H. B. Deising (Berlin; Heidelberg: Springer Berlin Heidelberg), 337-361.

Kuhl, C., Tautenhahn, R., Böttcher, C., Larson, T. R., and Neumann, S. (2012). CAMERA: an integrated strategy for compound spectra extraction and annotation of liquid chromatography/mass spectrometry data sets. Anal. Chem. 84, 283-289. doi: 10.1021/ac202450g

Lassowskat, I., Naumann, K., Lee, J., and Scheel, D. (2013). PAPE (PrefractionationAssisted Phosphoprotein Enrichment): a novel approach for Phosphoproteomic analysis of green tissues from plants. Proteomes 1, 254-274. doi: 10.3390/proteomes 1030254

Lee, J., Rudd, J. J., Macioszek, V. K., and Scheel, D. (2004). Dynamic changes in the localization of MAPK cascade components controlling pathogenesis-related 
$(P R)$ gene expression during innate immunity in parsley. J. Biol. Chem. 279, 22440-22448. doi: 10.1074/jbc.M401099200

Liese, A., and Romeis, T. (2013). Biochemical regulation of in vivo function of plant calcium-dependent protein kinases (CDPK). Biochim. Biophys. Acta 1833, 1582-1589. doi: 10.1016/j.bbamcr.2012.10.024

Ligterink, W., Kroj, T., Zurnieden, U., Hirt, H., and Scheel, D. (1997). Receptormediated activation of a MAP kinase in pathogen defense of plants. Science 276, 2054-2057. doi: 10.1126/science.276.5321.2054

Liu, Y., Ren, D., Pike, S., Pallardy, S., Gassmann, W., and Zhang, S. (2007). Chloroplast-generated reactive oxygen species are involved in hypersensitive response-like cell death mediated by a mitogen-activated protein kinase cascade. Plant J. 51, 941-954. doi: 10.1111/j.1365-313X.2007.03191.x

Liu, Y., and Zhang, S. (2004). Phosphorylation of 1-aminocyclopropane-1carboxylic acid synthase by MPK6, a stress-responsive mitogen-activated protein kinase, induces ethylene biosynthesis in Arabidopsis. Plant Cell 16, 3386-3399. doi: 10.1105/tpc.104.026609

Maldonado-Bonilla, L. D., Eschen-Lippold, L., Gago-Zachert, S., Tabassum, N., Bauer, N., Scheel, D., et al. (2014). The Arabidopsis tandem zinc finger 9 protein binds RNA and mediates pathogen-associated molecular pattern-triggered immune responses. Plant Cell Physiol. 55, 412-425. doi: 10.1093/pcp/pct175

Mao, G., Meng, X., Liu, Y., Zheng, Z., Chen, Z., and Zhang, S. (2011). Phosphorylation of a WRKY transcription factor by two pathogen-responsive MAPKs drives phytoalexin biosynthesis in Arabidopsis. Plant Cell 23, 1639-1653. doi: 10.1105/tpc.111.084996

Meng, X., Xu, J., He, Y., Yang, K. Y., Mordorski, B., Liu, Y., et al. (2013). Phosphorylation of an ERF transcription factor by Arabidopsis MPK3/MPK6 regulates plant defense gene induction and fungal resistance. Plant Cell 25, 1126-1142. doi: 10.1105/tpc.112.109074

Meng, X., and Zhang, S. (2013). MAPK cascades in plant disease resistance signaling. Annu. Rev. Phytopathol. 51, 245-266. doi: 10.1146/annurev-phyto082712-102314

Merkouropoulos, G., Andreasson, E., Hess, D., Boller, T., and Peck, S. C. (2008) An Arabidopsis protein phosphorylated in response to microbial elicitation, AtPHOS32, is a substrate of MAP kinases 3 and 6. J. Biol. Chem. 283, 10493-10499. doi: 10.1074/jbc.M800735200

Mersmann, S., Bourdais, G., Rietz, S., and Robatzek, S. (2010). Ethylene signalling regulates accumulation of the FLS2 receptor and is required for the oxidative burst contributing to plant immunity. Plant Physiol. 154, 391-400. doi 10.1104/pp.110.154567

Miles, G. P., Samuel, M. A., Zhang, Y., and Ellis, B. E. (2005). RNA interferencebased (RNAi) suppression of AtMPK6, an Arabidopsis mitogen-activated protein kinase, results in hypersensitivity to ozone and misregulation of AtMPK3. Environ. Pollut. 138, 230-237. doi: 10.1016/j.envpol.2005.04.017

Miller, G., Schlauch, K., Tam, R., Cortes, D., Torres, M. A., Shulaev, V., et al. (2009). The plant NADPH oxidase RBOHD mediates rapid systemic signaling in response to diverse stimuli. Sci. Signal. 2, ra45. doi: 10.1126/scisignal.2000448

Millet, Y. A., Danna, C. H., Clay, N. K., Songnuan, W., Simon, M. D., WerckReichhart, D., et al. (2010). Innate immune responses activated in Arabidopsis roots by microbe-associated molecular patterns. Plant Cell 22, 973-990. doi: 10.1105/tpc. 109.069658

Monaghan, J., and Zipfel, C. (2012). Plant pattern recognition receptor complexes at the plasma membrane. Curr. Opin. Plant Biol. 15, 349-357. doi: 10.1016/j.pbi.2012.05.006

Nafisi, M., Goregaoker, S., Botanga, C. J., Glawischnig, E., Olsen, C. E., Halkier, B. A., et al. (2007). Arabidopsis cytochrome P450 monooxygenase 71A13 catalyzes the conversion of indole-3-acetaldoxime in camalexin synthesis. Plant Cell 19, 2039-2052. doi: 10.1105/tpc.107.051383

Nicaise, V., Roux, M., and Zipfel, C. (2009). Recent advances in PAMP-triggered immunity against bacteria: pattern recognition receptors watch over and raise the alarm. Plant Physiol. 150, 1638-1647. doi: 10.1104/pp.109.139709

Nühse, T. S., Boller, T., and Peck, S. C. (2003). A plasma membrane syntaxin is phosphorylated in response to the bacterial elicitor flagellin. J. Biol. Chem. 278, 45248-45254. doi: 10.1074/jbc.M307443200

Nürnberger, T., Nennstiel, D., Jabs, T., Sacks, W. R., Hahlbrock, K., and Scheel, D. (1994). High affinity binding of a fungal oligopeptide elicitor to parsley plasma membranes triggers multiple defense responses. Cell 78, 449-460. doi: 10.1016/0092-8674(94)90423-5

Pecher, P., Eschen-Lippold, L., Herklotz, S., Kuhle, K., Naumann, K., Bethke, G., et al. (2014). The Arabidopsis thaliana mitogen-activated protein kinases MPK3 and MPK6 target a subclass of 'VQ-motif'-containing proteins to regulate immune responses. New Phytol. 203, 592-606. doi: 10.1111/nph.12817

Pitzschke, A., Schikora, A., and Hirt, H. (2009). MAPK cascade signalling networks in plant defence. Curr. Opin. Plant Biol. 12, 421-426. doi: 10.1016/j.pbi.2009.06.008

Popescu, S. C., Popescu, G. V., Bachan, S., Zhang, Z., Gerstein, M., Snyder, M., et al. (2009). MAPK target networks in Arabidopsis thaliana revealed using functional protein microarrays. Genes Dev. 23, 80-92. doi: 10.1101/gad.1740009

Qiu, J. L., Fiil, B. K., Petersen, K., Nielsen, H. B., Botanga, C. J., Thorgrimsen, S., et al. (2008). Arabidopsis MAP kinase 4 regulates gene expression through transcription factor release in the nucleus. EMBO J. 27, 2214-2221. doi: 10.1038/emboj.2008.147

Ranf, S., Eschen-Lippold, L., Pecher, P., Lee, J., and Scheel, D. (2011). Interplay between calcium signalling and early signalling elements during defence responses to microbe- or damage-associated molecular patterns. Plant J. 68, 100-113. doi: 10.1111/j.1365-313X.2011.04671.x

Ren, D., Yang, H., and Zhang, S. (2002). Cell death mediated by MAPK is associated with hydrogen peroxide production in Arabidopsis. J. Biol. Chem. 277, 559-565. doi: 10.1074/jbc.M109495200

Schenke, D., Böttcher, C., and Scheel, D. (2011). Crosstalk between abiotic ultraviolet-B stress and biotic (flg22) stress signalling in Arabidopsis prevents flavonol accumulation in favor of pathogen defence compound production. Plant Cell Environ. 34, 1849-1864. doi: 10.1111/j.1365-3040.2011.02381.x

Schulz, P., Herde, M., and Romeis, T. (2013). Calcium-dependent protein kinases: hubs in plant stress signaling and development. Plant Physiol. 163, 523-530. doi: 10.1104/pp.113.222539

Schweizer, F., Fernandez-Calvo, P., Zander, M., Diez-Diaz, M., Fonseca, S., Glauser, G., et al. (2013). Arabidopsis basic helix-loop-helix transcription factors MYC2, MYC3, and MYC4 regulate glucosinolate biosynthesis, insect performance, and feeding behavior. Plant Cell 25, 3117-3132. doi: 10.1105/tpc.113.115139

Smith, C. A., Want, E. J., O’maille, G., Abagyan, R., and Siuzdak, G. (2006). XCMS: processing mass spectrometry data for metabolite profiling using nonlinear peak alignment, matching, and identification. Anal. Chem. 78, 779-787. doi: $10.1021 / \mathrm{ac} 051437 \mathrm{y}$

Stauber, E. J., Kuczka, P., van Ohlen, M., Vogt, B., Janowitz, T., Piotrowski, M., et al. (2012). Turning the 'mustard oil bomb' into a 'cyanide bomb': aromatic glucosinolate metabolism in a specialist insect herbivore. PLoS ONE 7:e35545. doi: 10.1371/journal.pone.0035545

Stein, M., Dittgen, J., Sanchez-Rodriguez, C., Hou, B. H., Molina, A., SchulzeLefert, P., et al. (2006). Arabidopsis PEN3/PDR8, an ATP binding cassette transporter, contributes to nonhost resistance to inappropriate pathogens that enter by direct penetration. Plant Cell 18, 731-746. doi: 10.1105/tpc.105.038372

Tena, G., Boudsocq, M., and Sheen, J. (2011). Protein kinase signaling networks in plant innate immunity. Curr. Opin. Plant Biol. 14, 519-529. doi: 10.1016/j.pbi.2011.05.006

Torres, M. A., Dangl, J. L., and Jones, J. D. (2002). Arabidopsis gp91phox homologues AtrbohD and AtrbohF are required for accumulation of reactive oxygen intermediates in the plant defense response. Proc. Natl. Acad. Sci. U.S.A. 99, 517-522. doi: 10.1073/pnas.01245249

Tsuda, K., Mine, A., Bethke, G., Igarashi, D., Botanga, C. J., Tsuda, Y., et al. (2013). Dual regulation of gene expression mediated by extended MAPK activation and salicylic acid contributes to robust innate immunity in Arabidopsis thaliana. PLoS Genet. 9:e1004015. doi: 10.1371/journal.pgen.1004015

Vizcaíno, J. A., Deutsch, E. W., Wang, R., Csordas, A., Reisinger, F., Rios, D., et al. (2014). ProteomeXchange provides globally coordinated proteomics data submission and dissemination. Nat. Biotechnol. 32, 223-226. doi: 10.1038/ nbt. 2839

Wang, H., Liu, Y., Bruffett, K., Lee, J., Hause, G., Walker, J. C., et al. (2008). Haploinsufficiency of MPK3 in MPK6 mutant background uncovers a novel function of these two MAPKs in Arabidopsis ovule development. Plant Cell 20, 602-613. doi: 10.1105/tpc.108.058032

Wang, H., Ngwenyama, N., Liu, Y., Walker, J. C., and Zhang, S. (2007). Stomatal development and patterning are regulated by environmentally responsive mitogen-activated protein kinases in Arabidopsis. Plant Cell 19, 63-73. doi: 10.1105/tpc.106.048298

Wang, H.-C., Wu, J.-S., Chia, J.-C., Yang, C.-C., Wu, Y.-J., and Juang, R.-H. (2009). Phytochelatin Synthase is regulated by protein phosphorylation at a threonine residue near its catalytic site. J. Agric. Food Chem. 57, 7348-7355. doi: 10.1021/jf9020152 
Wang, P., Du, Y., Li, Y., Ren, D., and Song, C. P. (2010). Hydrogen peroxidemediated activation of MAP kinase 6 modulates nitric oxide biosynthesis and signal transduction in Arabidopsis. Plant Cell 22, 2981-2998. doi: 10.1105/tpc.109.072959

Winde, I., and Wittstock, U. (2011). Insect herbivore counteradaptations to the plant glucosinolate-myrosinase system. Phytochemistry 72, 1566-1575. doi: 10.1016/j.phytochem.2011.01.016

Wolschin, F., and Weckwerth, W. (2005). Combining metal oxide affinity chromatography (MOAC) and selective mass spectrometry for robust identification of in vivo protein phosphorylation sites. Plant Methods 1:9. doi: 10.1186/17464811-1-9

Xu, J., and Chua, N. H. (2010). Processing bodies and plant development. Curr. Opin. Plant Biol. 14, 88-93. doi: 10.1016/j.pbi.2010.10.003

$\mathrm{Xu}$, J., and Chua, N.-H. (2012). Dehydration stress activates Arabidopsis MPK6 to signal DCP1 phosphorylation. EMBO J. 31, 1975-1984. doi: 10.1038/emboj.2012.56

Zipfel, C. (2009). Early molecular events in PAMP-triggered immunity. Curr. Opin. Plant Biol. 12, 414-420. doi: 10.1016/j.pbi.2009.06.003
Conflict of Interest Statement: The authors declare that the research was conducted in the absence of any commercial or financial relationships that could be construed as a potential conflict of interest.

Received: 17 July 2014; accepted: 27 September 2014; published online: 20 October 2014.

Citation: Lassowskat I, Böttcher C, Eschen-Lippold L, Scheel D and Lee J (2014) Sustained mitogen-activated protein kinase activation reprograms defense metabolism and phosphoprotein profile in Arabidopsis thaliana. Front. Plant Sci. 5:554. doi: $10.3389 / \mathrm{fpls} .2014 .00554$

This article was submitted to Plant Proteomics, a section of the journal Frontiers in Plant Science.

Copyright (C) 2014 Lassowskat, Böttcher, Eschen-Lippold, Scheel and Lee. This is an open-access article distributed under the terms of the Creative Commons Attribution License (CC BY). The use, distribution or reproduction in other forums is permitted, provided the original author(s) or licensor are credited and that the original publication in this journal is cited, in accordance with accepted academic practice. No use, distribution or reproduction is permitted which does not comply with these terms. 